\title{
Reduction of one-loop integrals with higher poles by unitarity cut method
}

\author{
Bo Feng ${ }^{a, b, c}$ and Hongbin Wang ${ }^{a, 1}$ \\ ${ }^{a}$ Zhejiang Institute of Modern Physics, Zhejiang University, \\ Hangzhou, 310027, P.R. China \\ ${ }^{b}$ Center of Mathematical Science, Zhejiang University, \\ Hangzhou, 31002\%, P.R. China \\ ${ }^{c}$ Peng Huanwu Center for Fundamental Theory, \\ Hefei, Anhui 230026, China \\ E-mail: fengbo@zju.edu.cn, 21836003@zju.edu.cn
}

ABSTRACT: Unitarity cut method has been proved to be very useful in the computation of one-loop integrals. In this paper, we generalize the method to the situation where the powers of propagators in the denominator are larger than one in general. We show how to use the trick of differentiation over masses to translate the problem to the integrals where all powers are just one. Then by using the unitarity cut method, we can find the wanted reduction coefficients of all basis except the tadpole. Using this method, we calculate the reduction of scalar bubble, scalar triangle, scalar box and scalar pentagon with general power of propagators.

KEYworDS: Scattering Amplitudes, Renormalization Regularization and Renormalons ArXiv EPRINT: 2104.00922

\footnotetext{
${ }^{1}$ Corresponding author.
} 


\section{Contents}

1 Motivation 1

2 Bubble 3

2.1 Recurrence relation $\quad 6$

3 Triangle $r$

3.1 Recurrence relation 9

3.2 Triangle $(1,1,2) \quad 12$

3.2.1 Cut $K_{1} \quad 12$

3.2.2 Cut $K_{2} \quad 13$

3.2.3 Cut $K_{3} \quad 14$

3.2.4 A short summary 15

4 Box $\quad 15$

$\begin{array}{llr}4.1 & \text { Box } I_{4}(1,1,1,2) & 17\end{array}$

5 Pentagon $\quad 20$

5.1 Cut $K_{1}$ of $I_{2}(1,1,1,1,2) \quad 23$

$\begin{array}{llr}6 & \text { Conclusion } & 29\end{array}$

\section{Motivation}

Unitarity cut method [1-3] has been proved to be very efficient method to calculate oneloop amplitudes. The power of this method is that using the holomorphic anomaly [4], one can analytically carry out the reduced phase space integration with double cuts, thus to extract the wanted reduction coefficients of master basis. The phase space integration method has been systematically developed for pure $4 D$-dimension in $[5,6]$ and for general $(4-2 \epsilon)$-dimension in [7-9]. With these results, pure analytic algebraic expressions for reduction coefficients have been given in [9-11] and their properties have been studied in $[12,13]$.

All above works have been assumed that the power of propagators in the denominator of one-loop integrals is just one. This assumption is harmless for general situations, but higher power of propagators does appear in some situation, ${ }^{1}$ for example, in higher loops or in the middle steps if one use the IBP method to do the reduction. Thus it is naturally to ask if the unitarity cut method can be applied to these more general situations.

\footnotetext{
${ }^{1}$ Some works for the higher power of propagators can be found in [14-16].
} 
In this paper, we consider the reduction of one-loop integrals with general pole structures, i.e., propagators could have general power. The general integration will be given by

$$
\mathcal{M}[\ell] \equiv \int \frac{d^{D} \ell}{(2 \pi)^{D / 2}} \frac{\mathcal{N}[\ell]}{\prod_{j=1}^{n}\left(\left(\ell-K_{j}\right)^{2}-m_{j}^{2}+i \epsilon\right)^{a_{j}}}, \quad a_{i} \geq 1
$$

where $\mathcal{N}[\ell]$ is an arbitrary polynomial function of $\ell$. According to the PV-reduction method [17], we can decompose

$$
\mathcal{M}[\ell]=\sum_{t} c_{t} \mathcal{I}_{t}[\ell]
$$

where $c_{t}$ 's are the rational functions and $\mathcal{I}_{t}$ are scalar basis of tadpole, bubble, triangle, box and pentagon. Let us consider the $c_{i}$ 's first. The data entering the integral (1.1) are the external momenta, the masses and the polarization vectors (the coupling constants are overall factor, so can be dropped in the discussion). The $c_{i}$ should be the rational functions of these data with Lorentz invariant contractions.

The idea of unitarity cut method is to compare the imaginary part of both sides of (1.2). Since $c_{t}$ 's are rational functions without imaginary contributions, we have

$$
\operatorname{Im}(\mathcal{M}[\ell])=\sum_{t} c_{t} \operatorname{Im}\left(\mathcal{I}_{t}[\ell]\right)
$$

Different master integrals have different analytic structures for the imaginary part (we will call them as the "signature"), thus if we can analytically computer the left hand side, we can do the spliting according to the analytic signature of each master integral and find expansion coefficients $c_{t}$ at the right hand side. With this thought, the key of reduction by unitarity cut method is to compute the imaginary part of $\mathcal{M}[\ell]$. When all $a_{j}=1$, the computation of left hand side (1.3) is transformed to the reduced phase space integration with double cuts, which we know how to do it as reviewed in the first paragraph of this section. For general $a_{i}$, we use following trick to solve the problem. Noticing that ${ }^{2}$

$$
\begin{aligned}
& \int \frac{d^{D} \ell}{(2 \pi)^{D / 2}} \frac{\mathcal{N}[\ell]}{\prod_{j=1}^{n}\left(\left(\ell-K_{j}\right)^{2}-m_{j}^{2}+i \epsilon\right)^{a_{i}}} \\
& \quad=\left.\left\{\prod_{j=1}^{n} \frac{1}{\left(a_{j}-1\right) !} \frac{d^{a_{j}-1}}{d \eta_{j}^{a_{j}-1}} \int \frac{d^{D} \ell}{(2 \pi)^{D / 2}} \frac{\mathcal{N}[\ell]}{\prod_{j=1}^{n}\left(\left(\ell-K_{j}\right)^{2}-m_{j}^{2}-\eta_{j}+i \epsilon\right)}\right\}\right|_{\eta_{j} \rightarrow 0}
\end{aligned}
$$

the computation of the imaginary part of an one-loop integral is transformed to the reduced phase space integration with double cut for the case with all $a_{i}=1$. More explicitly, let us separate both sides of (1.4) to the real and imaginary part, we have

$$
\operatorname{Re}[L]+i \operatorname{Im}[L]=\left\{\prod_{j=1}^{n} \frac{1}{\left(a_{j}-1\right) !} \frac{d^{a_{j}-1}}{d \eta_{j}^{a_{j}-1}}(\operatorname{Re}[R]+i \operatorname{Im}[R])\right\} \mid \eta_{\eta_{j} \rightarrow 0}
$$

\footnotetext{
${ }^{2}$ The same trick has also been used to get the homogenous solution of differential equations by maximum cut in [18].
} 
Since the $\eta_{i}$ 's are defined to take real values, we have

$\operatorname{Re}[L]+i \operatorname{Im}[L]=\left.\left\{\prod_{j=1}^{n} \frac{1}{\left(a_{j}-1\right) !} \frac{d^{a_{j}-1}}{d \eta_{j}^{a_{j}-1}} \operatorname{Re}[R]\right\}\right|_{\eta_{j} \rightarrow 0}+\left.i\left\{\prod_{j=1}^{n} \frac{1}{\left(a_{j}-1\right) !} \frac{d^{a_{j}-1}}{d \eta_{j}^{a_{j}-1}} \operatorname{Im}[R]\right\}\right|_{\eta_{j} \rightarrow 0}$

thus we get

$$
\operatorname{Im}[L]=\left.\left\{\prod_{j=1}^{n} \frac{1}{\left(a_{j}-1\right) !} \frac{d^{a_{j}-1}}{d \eta_{j}^{a_{j}-1}} \operatorname{Im}[R]\right\}\right|_{\eta_{j} \rightarrow 0}
$$

For general $\mathcal{N}[\ell]$ in $(1.4)$, we know the expansion

$$
\operatorname{Im}[R]=\sum_{t} c_{t} \operatorname{Im}\left(\mathcal{I}_{t}[\ell]\right)
$$

and the action of $\frac{d}{d \eta}$ will act on both $c_{t}$ and $\operatorname{Im}\left(\mathcal{I}_{t}[\ell]\right)$. Since the function $c_{t}$ 's have been given in [9-11], the unknown piece is the action of $\frac{d}{d \eta}$ on $\operatorname{Im}\left(\mathcal{I}_{t}[\ell]\right)$ and its expansion. In another words, we just need to consider the reduction of general power with $\mathcal{N}[\ell]=1$ in (1.1) for $n \leq 5$.

The plan of the paper is following. In the section two, we consider the reduction of bubbles with higher poles. We establish the general recurrence relation and check our results with some examples. Same method has been applied to triangles, boxes and pentagons in the section three, four and five. A brief conclusion is given in the section six.

\section{Bubble}

For bubble topology, let us define

$$
I_{2}(a, b)\left[K ; M_{1}, M_{2}\right] \equiv \int \frac{d^{4-2 \epsilon} p}{(2 \pi)^{4-2 \epsilon}} \frac{1}{\left(p^{2}-M_{1}^{2}\right)^{a}\left((p-K)^{2}-M_{2}^{2}\right)^{b}}
$$

to be the general scalar bubble integral with higher power of propagators. The master integral of bubble is the case $n=m=1$ and for this special case, sometimes we just write it as $\mathcal{I}_{2}\left[K ; M_{1}, M_{2}\right]$ or just $\mathcal{I}_{2}$ for simplicity. The reduction of $I_{2}(a, b)\left[K ; M_{1}, M_{2}\right]$ will be the following expansion

$$
I_{2}(a, b)\left[K ; M_{1}, M_{2}\right]=c_{2 \rightarrow 2}(a, b) \mathcal{I}_{2}\left[K ; M_{1}, M_{2}\right]+\sum_{i=1}^{2} c_{2 \rightarrow 1 ; i}(a, b) \mathcal{I}_{1 ; i}\left[M_{i}\right]
$$

where $c_{2 \rightarrow 1 ; i}$ means that when reducing the bubble to the tadpole, the $i$-th propagator has been kept. The tadpole $\mathcal{I}_{1 ; i}\left[M_{i}\right]$ should be written in the standard form with the proper momentum shifting, i.e.,

$$
\mathcal{I}_{1 ; i}\left[M_{i}\right] \equiv \int \frac{d^{4-2 \epsilon} p}{(2 \pi)^{4-2 \epsilon}} \frac{1}{\left(p^{2}-M_{i}^{2}\right)}
$$

Expansion coefficients in (2.2) can be found by various methods, for example, the IBP method. However, in this paper, we will try to use the unitarity cut method to find 
expansion coefficients. When we use the unitarity cut method, the tadpole can not be detected, thus tadpole coefficients can not be found by this way. Although we will not consider the tadpole coefficients in this paper, we want to point out that some efforts have been done to fill the gap by using the single cut [19-21].

The unitarity cut of $I_{2}(1,1)$ is given by (see references $\left.[7-9]\right)^{3}$

$$
\mathcal{C}\left[\mathcal{I}_{2}\right]=\left(K^{2}\right)^{-1+\epsilon} \Delta^{\frac{1}{2}-\epsilon} \int_{0}^{1} \mathrm{~d} u u^{-1-\epsilon} \sqrt{1-u}
$$

where

$$
\begin{aligned}
\Delta\left[K ; M_{1}, M_{2}\right] & =\left(K^{2}\right)^{2}+\left(M_{1}^{2}\right)^{2}+\left(M_{2}^{2}\right)^{2}-2 M_{1}^{2} M_{2}^{2}-2 K^{2} M_{1}^{2}-2 K^{2} M_{2}^{2} \\
& =-4 M_{1}^{2} M_{2}^{2}\left|\begin{array}{cc}
1 & -\frac{K^{2}-M_{1}^{2}-M_{2}^{2}}{2 M_{1} M_{2}} \\
-\frac{K^{2}-M_{1}^{2}-M_{2}^{2}}{2 M_{1} M_{2}} & 1
\end{array}\right|,
\end{aligned}
$$

which is the Landau surface of bubble of the first type of singularities. For the later convenience, let us define

$$
B u b^{(n)}=\frac{1}{2(n-\epsilon)} \int_{0}^{1} d u \frac{u^{n-\epsilon}}{\sqrt{1-u}}=\int_{0}^{1} \mathrm{~d} u u^{-1-\epsilon} u^{n} \sqrt{1-u} .
$$

Thus by comparing (2.6) with (2.4) we see that $\mathcal{C}\left[\mathcal{I}_{2}\right]=\left(K^{2}\right)^{-1+\epsilon} \Delta^{\frac{1}{2}-\epsilon} B u b^{(0)}$. The $B u b^{(n)}$ is well defined for $n \geq 0$ and it is easy to derive a recursion relation by integration-by-part

$$
B u b^{(n)}=\frac{(n-1-\epsilon)}{\left(n+\frac{1}{2}-\epsilon\right)} B u b^{(n-1)} .
$$

Solving it we get

$$
B u b^{(n)}=F^{(n)} B u b^{(0)}, \quad F^{(n)}=\frac{\Gamma(3 / 2-\epsilon) \Gamma(n-\epsilon)}{\Gamma(-\epsilon) \Gamma(n+3 / 2-\epsilon)} .
$$

We find that when doing the reduction for triangles and boxes, we will meet the form $B u b^{(n)}$ with the negative integer $n$. For this case, we can use (2.7) to analytically continue from positive $n$ to negative $n$. For example, using $n=0$ in (2.7), we get

$$
B u b^{(-1)}=\frac{(1-2 \epsilon)}{2(-1-\epsilon)} B u b^{(0)}
$$

Having above preparation, now we consider the reduction of $I_{2}(a, b)$. According to our general idea in (1.7), we should calculate ${ }^{4}\left(\frac{\partial}{\partial M_{1}^{2}}\right)^{a-1}\left(\frac{\partial}{\partial M_{2}^{2}}\right)^{b-1} \Delta^{\frac{1}{2}-\epsilon}$. To get a better idea, let us start with $\left(\frac{\partial}{\partial M_{1}^{2}}\right)^{n} \Delta^{\frac{1}{2}-\epsilon}$. Before doing this, we rewrite $\Delta$ as

$$
\Delta=\left(M_{1}^{2}-M_{2}^{2}-K^{2}-\sqrt{2 K^{2} M_{2}^{2}}\right)\left(M_{1}^{2}-M_{2}^{2}-K^{2}+\sqrt{2 K^{2} M_{2}^{2}}\right)
$$

\footnotetext{
${ }^{3}$ In the reference [9] when we do the general one-loop unitarity cut phase space integration, an overall factor $\left(\frac{\Delta\left[K, M_{1}, M_{2}\right]}{K^{2}}\right)^{-\epsilon}$ has been neglected in later computation (see the equation after eq. (9)). This is fine for the work in [9], but since it depends on the masses, it is crucial for current computation and we must include it back.

${ }^{4}$ From the discussion of (1.4) one can see that the role of $\eta$ is identical with $m^{2}$. For scalar basis, $m$ does not appear in other places, so we can take the derivative of $m^{2}$ instead of $\eta$ without making any mistake.
} 
where each factor is linear in $M_{1}^{2}$. It is easy to get the $n$-th derivative of $M_{1}^{2}$ is given by

$$
\begin{aligned}
\left(\frac{\partial}{\partial M_{1}^{2}}\right)^{n} \Delta^{\frac{1}{2}-\epsilon}= & \sum_{\lambda=0}^{n} C_{n}^{\lambda}\left(\frac{\partial}{\partial M_{1}^{2}}\right)^{n-\lambda}\left\{\left(M_{1}^{2}-M_{2}^{2}-K^{2}-\sqrt{2 K^{2} M_{2}^{2}}\right)^{\frac{1}{2}-\epsilon}\right\} \\
& \times\left(\frac{\partial}{\partial M_{1}^{2}}\right)^{\lambda}\left\{\left(M_{1}^{2}-M_{2}^{2}-K^{2}+\sqrt{2 K^{2} M_{2}^{2}}\right)^{\frac{1}{2}-\epsilon}\right\}
\end{aligned}
$$

Using $\Gamma(x+1)=x \Gamma(x)$, we have

$$
\frac{d^{n}(x-a)^{b}}{d x^{n}}=b(b-1) . .(b-n+1)(x-a)^{b-n}=\frac{\Gamma(b+1)}{\Gamma(b+1-n)}(x-a)^{b-n}
$$

thus $(2.11)$ is

$$
\left(\frac{\partial}{\partial M_{1}^{2}}\right)^{n} \Delta^{\frac{1}{2}-\epsilon}=\Delta^{\frac{1}{2}-\epsilon}\left\{\sum_{\lambda=0}^{n} C_{n}^{\lambda} \frac{\Gamma\left(\frac{3}{2}-\epsilon\right)}{\Gamma\left(\frac{3}{2}-\epsilon-(n-\lambda)\right)} \Delta_{M_{1},-}^{-(n-\lambda)} \frac{\Gamma\left(\frac{3}{2}-\epsilon\right)}{\Gamma\left(\frac{3}{2}-\epsilon-\lambda\right)} \Delta_{M_{1},+}^{-\lambda}\right\}
$$

where we have defined

$$
\Delta_{M_{1}, \pm}=M_{1}^{2}-M_{2}^{2}-K^{2} \pm \sqrt{2 K^{2} M_{2}^{2}}
$$

Using above result, we can find the reduction coefficient of $I_{2}(n+1,1)$ by $(1.7)$ since

$$
\mathcal{C}\left[I_{2}(n+1,1)\right]=\frac{1}{n !}\left(\frac{\partial}{\partial M_{1}^{2}}\right)^{n} \mathcal{C}\left[\mathcal{I}_{2}\right]=c_{2 \rightarrow 2}(n+1,1) \mathcal{C}\left[\mathcal{I}_{2}\right]
$$

where

$$
c_{2 \rightarrow 2}(n+1,1)\left[K ; M_{1}, M_{2}\right]=\frac{1}{n !}\left\{\sum_{\lambda=0}^{n} C_{n}^{\lambda} \frac{\Gamma\left(\frac{3}{2}-\epsilon\right)}{\Gamma\left(\frac{3}{2}-\epsilon-(n-\lambda)\right)} \Delta_{M_{1},-}^{-(n-\lambda)} \frac{\Gamma\left(\frac{3}{2}-\epsilon\right)}{\Gamma\left(\frac{3}{2}-\epsilon-\lambda\right)} \Delta_{M_{1},+}^{-\lambda}\right\}
$$

by the result $(2.13)$.

For the general bubble coefficients of the reduction of $I_{2}(n+1, m+1)$, using the relation

$$
\mathcal{C}\left[I_{2}(n+1, m+1)\right]=\frac{1}{m ! n !}\left(\frac{\partial}{\partial M_{2}^{2}}\right)^{m}\left(\frac{\partial}{\partial M_{1}^{2}}\right)^{n} \mathcal{C}\left[I_{2}(1,1)\right]
$$

we get reduction coefficient as

$$
c_{2 \rightarrow 2}(n+1, m+1)\left[K ; M_{1}, M_{2}\right]=\frac{1}{m ! n ! \Delta^{\frac{1}{2}-\epsilon}}\left(\frac{\partial}{\partial M_{2}^{2}}\right)^{m}\left(\frac{\partial}{\partial M_{1}^{2}}\right)^{n} \Delta^{\frac{1}{2}-\epsilon}
$$

The general analytic expression of $c_{2 \rightarrow 2}(n+1, m+1)$ will be complicated to write down.

Now we check the result (2.18). The first check is by the symmetry. Noticing that when shifting $p \rightarrow p+K$, the (2.1) becomes

$$
\int \frac{d^{4-2 \epsilon} p}{(2 \pi)^{4-2 \epsilon}} \frac{1}{\left(\left((p+K)^{2}-M_{1}^{2}\right)^{a}\left(p^{2}-M_{2}^{2}\right)^{b}\right.}
$$


thus we have $I_{2}(a, b)\left[K ; M_{1}, M_{2}\right]=I_{2}(b, a)\left[-K ; M_{2}, M_{1}\right]$. When doing reduction at both sides, we must have the bubble coefficient to be same, i.e,

$$
c_{2 \rightarrow 2}(n+1, m+1)\left[K ; M_{1}, M_{2}\right]=c_{2 \rightarrow 2}(m+1, n+1)\left[-K ; M_{2}, M_{1}\right]
$$

From the first line of (2.18), the $\Delta$ is invariant under the replacement $\left[K ; M_{1}, M_{2}\right] \rightarrow$ $\left[-K ; M_{2}, M_{1}\right]$, so it does satisfy the relation $(2.20)$.

The second check is following. For one loop massless integral (example 5.2 of [22])

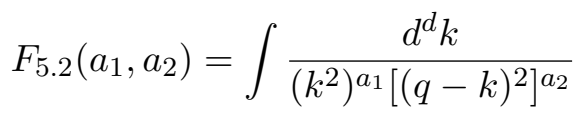

by the IBP method, one can derive the relation

$$
F\left(a_{1}, a_{2}>1\right)=-\frac{1}{\left(a_{2}-1\right) q^{2}}\left[\left(d-2 a_{1}-a_{2}+1\right) F\left(a_{1}, a_{2}-1\right)-\left(a_{2}-1\right) F\left(a_{1}-1, a_{2}\right)\right]
$$

and when $a_{2}=1$ we have

$$
F\left(a_{1}, 1\right)=-\frac{d-a_{1}-1}{\left(a_{1}-1\right) q^{2}} F\left(a_{1}-1,1\right) .
$$

To compare with our calculation, we should take $M_{1}, M_{2} \rightarrow 0, K \rightarrow q$ and $d \rightarrow 4-2 \epsilon$ after all derivatives related to $M_{1}^{2}, M_{2}^{2}$ having been done. We have used the Mathematica to check the massless case of our general result (2.18). The third check is that we have used the LiteRed [23] to explicitly calculate some examples with nonzero $M_{1}, M_{2}$ and we do find the match.

\subsection{Recurrence relation}

Like the IBP relation, the idea of (1.7) can be used to establish the recurrence relation for reduction coefficients. Let us start from the reduction of $I_{2}(a, 1)$. Noticing that

$$
\begin{aligned}
I_{2}(a, 1) & =\int \frac{d^{4-2 \epsilon} p}{(2 \pi)^{4-2 \epsilon}} \frac{1}{\left(p^{2}-M_{1}^{2}\right)^{a}\left((p-K)^{2}-M_{2}^{2}\right)} \\
& =\frac{1}{(a-1)} \frac{d}{d\left(M_{1}^{2}\right)} \frac{1}{(a-2) !} \frac{d^{a-2}}{d\left(M_{1}^{2}\right)^{a-2}} \int \frac{d^{4-2 \epsilon} p}{(2 \pi)^{4-2 \epsilon}} \frac{1}{\left(p^{2}-M_{1}^{2}\right)\left((p-K)^{2}-M_{2}^{2}\right)} \\
& =\frac{1}{(a-1)} \frac{d}{d\left(M_{1}^{2}\right)} I_{2}(a-1,1),
\end{aligned}
$$

if we know

$$
I_{2}(a-1,1)=c_{2 \rightarrow 2}(a-1,1)\left[K ; M_{1}, M_{2}\right] I_{2}(1,1)+\ldots
$$

where ... is for the tadpole part, we can write

$$
I_{2}(a, 1)=\left(\frac{1}{(a-1)} \frac{d c_{2 \rightarrow 2}(a-1,1)}{d\left(M_{1}^{2}\right)}\right) I_{2}(1,1)+\frac{c_{2 \rightarrow 2}(a-1,1)}{(a-1)} I_{2}(2,1)+\ldots
$$

where for simplicity we have dropped the dependence of $K, M$. From (2.26), we can read out a recurrence relation for the coefficient ${ }^{5}$

$$
c_{2 \rightarrow 2}(a, 1)\left[K ; M_{1}, M_{2}\right]=\left(\frac{1}{(a-1)} \frac{d c_{2 \rightarrow 2}(a-1,1)}{d\left(M_{1}^{2}\right)}\right)+\frac{c_{2 \rightarrow 2}(a-1,1)}{(a-1)} c_{2 \rightarrow 2}(2,1)
$$

\footnotetext{
${ }^{5}$ The relation $(2.27)$ holds for $a \geq 2$, but when $a=2$, the $c_{2 \rightarrow 2}(1,1)=1$ and the identity is trivial.
} 
Relation (2.27) tell us that staring from $c_{2 \rightarrow 2}(2,1)$, we can write down all $c_{2 \rightarrow 2}(a, 1)$.

The same idea can be used to write down the recurrence relation of general $c_{2 \rightarrow 2}(a, b)$. Noticing that

$$
\begin{aligned}
I_{2}(a, b) & ==\frac{1}{(b-1) !} \frac{d^{b-1}}{d\left(M_{2}^{2}\right)^{b-1}} I_{2}(a, 1)=\frac{1}{(b-1) !} \frac{d^{b-1}}{d\left(M_{2}^{2}\right)^{b-1}}\left\{c_{2 \rightarrow 2}(a, 1) I_{2}(1,1)+\ldots\right\} \\
& =\frac{1}{(b-1) !} \sum_{t=0}^{b-1} C_{b-1}^{t} \frac{d^{t} c_{2 \rightarrow 2}(a, 1)}{d\left(M_{2}^{2}\right)^{t}}(b-1-t) ! I_{2}(1, b-t)+\ldots
\end{aligned}
$$

we get immediately the recurrence relation

$$
c_{2 \rightarrow 2}(a, b)=\frac{1}{(b-1) !} \sum_{t=0}^{b-1} C_{b-1}^{t}(b-1-t) ! c_{2 \rightarrow 2}(1, b-t) \frac{d^{t} c_{2 \rightarrow 2}(a, 1)}{d\left(M_{2}^{2}\right)^{t}}
$$

where $c_{2 \rightarrow 2}(1, m)$ can be obtained from $c_{2 \rightarrow 2}(m, 1)$ by proper replacement as given in $(2.20)$. Result (2.29) shows that knowing the coefficient $c_{2 \rightarrow 2}(2,1)$ is enough for general reduction coefficients. The similar idea can be used to the reduction for triangle, box and pentagon as will be shown later.

\section{Triangle}

For triangle topology, let us define ${ }^{6}$

$$
\begin{aligned}
& I_{3}\left(n_{1}, n_{2}, n_{3}\right)\left[K_{1}, K_{2}, K_{3} ; M_{1}, M_{2}, m_{1}\right] \\
& \quad=\int \frac{d^{4-2 \epsilon} p}{(2 \pi)^{4-2 \epsilon}} \frac{1}{\left(p^{2}-M_{1}^{2}\right)^{n_{1}}\left(\left(p-K_{1}\right)^{2}-M_{2}^{2}\right)^{n_{2}}\left(\left(p+K_{3}\right)^{2}-m_{1}^{2}\right)^{n_{3}}}
\end{aligned}
$$

For the special case, i.e., $n_{1}=n_{2}=n_{3}=1$, we get the familiar scalar triangle basis, which for simplicity, we will denote as $\mathcal{I}_{3}\left[K_{1}, K_{2}, K_{3} ; M_{1}, M_{2}, m_{1}\right]$. The unitarity cut of $\mathcal{I}_{3}$ with the cut momentum $K_{1}$ has been given by [9]

$$
\mathcal{C}\left(I_{3}\right)=-\left(\frac{4 K_{1}^{2}}{\Delta\left[K_{1}, M_{1}, M_{2}\right]}\right)^{\epsilon} \frac{1}{\sqrt{\Delta_{3 ; m=0}}} \operatorname{Tri}^{(0)}(Z)
$$

with

$\operatorname{Tri}^{(n)}(Z)=\int_{0}^{1} d u u^{-1-\epsilon} u^{n} \ln \left(\frac{Z+\sqrt{1-u}}{Z-\sqrt{1-u}}\right)=\frac{-Z}{n-\epsilon} \int_{0}^{1} d u \frac{u^{n-\epsilon}}{\sqrt{1-u}\left(1-u-Z^{2}\right)}, \quad n \geq 0$

where $Z$ is given by

$$
Z=-\frac{\left(2 K_{1} \cdot Q\right) K_{1}^{2}}{\sqrt{\Delta_{3 ; m=0} \Delta\left[K_{1}, M_{1}, M_{2}\right]}}
$$

\footnotetext{
${ }^{6}$ Although by momentum conservation, we will have $K_{1}+K_{2}+K_{3}=0$. Keeping them all in the parameter will make the symmetry more transparent.
} 
with $^{7}$

$$
\begin{aligned}
\Delta_{3 ; m=0} & =4\left(\left(K_{1} \cdot K_{3}\right)^{2}-K_{1}^{2} K_{3}^{2}\right)=-4 \operatorname{det}\left(\begin{array}{cc}
K_{1}^{2} & K_{1} \cdot K_{3} \\
K_{1} \cdot K_{3} & K_{3}^{2}
\end{array}\right) \\
\Delta\left[K_{1}, M_{1}, M_{2}\right] & =\left(K_{1}^{2}\right)^{2}+\left(M_{1}^{2}\right)^{2}+\left(M_{2}^{2}\right)^{2}-2 K_{1}^{2} M_{1}^{2}-2 K_{1}^{2} M_{2}^{2}-2 M_{1}^{2} M_{2}^{2} \\
K_{1} \cdot Q & =\frac{K_{1}^{2}+M_{1}^{2}-M_{2}^{2}}{K_{1}^{2}}\left(K_{1} \cdot K_{3}\right)+\left(K_{3}^{2}+M_{1}^{2}-m_{1}^{2}\right)
\end{aligned}
$$

The $\operatorname{Tri}^{(n)}(Z)$ has the recurrence relation $[8,9]$

$$
\operatorname{Tri}^{(n)}(Z)=-\frac{\left(Z^{2}-1\right)(n-1-\epsilon)}{n-\epsilon} \operatorname{Tri}^{(n-1)}(Z)+\frac{2 Z(n-1-\epsilon)}{(n-\epsilon)} B u b^{(n-1)}
$$

which will be useful later.

For general $n_{i}$, the $I_{3}\left(n_{1}, n_{2}, n_{3}\right)$ will be reduced to the sum of one triangle $I_{3}$, three bubbles $I_{2 ; \bar{i}}, i=1,2,3$ (where $\bar{i}$ means to set $n_{i}=0$ in (3.1) and other $n_{j}$ 's one) and three tadpoles $I_{1 ; i}, i=1,2,3$ (where $i$ means to set $n_{i}=1$ in (3.1) and other $n_{j}$ 's zero). In other words, we will have the expansion

$$
I_{3}\left(n_{1}, n_{2}, n_{3}\right)=c_{3 \rightarrow 3}\left(n_{1}, n_{2}, n_{3}\right) \mathcal{I}_{3}+\sum_{i=1}^{3} c_{3 \rightarrow 2 ; i}\left(n_{1}, n_{2}, n_{3}\right) \mathcal{I}_{2 ; i}+\sum_{i=1}^{3} c_{3 \rightarrow 1 ; i}\left(n_{1}, n_{2}, n_{3}\right) \mathcal{I}_{1 ; i}
$$

where for simplicity we have dropped the dependence over $K, M$ of coefficients $c$ 's. We want to emphasize that when writing down $\mathcal{I}_{2 ; \bar{i}}$, we need to do the proper momentum shifting to reach the standard form (2.1). More explicitly, we will have

$$
\mathcal{I}_{2 ; \overline{3}}=\int \frac{d^{4-2 \epsilon} p}{(2 \pi)^{4-2 \epsilon}} \frac{1}{\left(p^{2}-M_{1}^{2}\right)\left(\left(p-K_{1}\right)^{2}-M_{2}^{2}\right)}
$$

and

$$
\mathcal{I}_{2 ; \overline{1}}=\int \frac{d^{4-2 \epsilon} p}{(2 \pi)^{4-2 \epsilon}} \frac{1}{\left(p^{2}-M_{2}^{2}\right)\left(\left(p-K_{2}\right)^{2}-m_{1}^{2}\right)}
$$

by using the shifting $p \rightarrow p+K_{1}$, and finally

$$
\mathcal{I}_{2 ; \overline{2}}=\int \frac{d^{4-2 \epsilon} p^{4}}{(2 \pi)^{4-2 \epsilon}} \frac{1}{\left(\left(p-K_{3}\right)^{2}-M_{1}^{2}\right)\left(p^{2}-m_{1}^{2}\right)}
$$

by using the shifting $p \rightarrow p+K_{1}+K_{2}$. For later convenience, let us give a more general bubbles coming from the triangle reduction (3.1)

$$
\begin{aligned}
I_{2 ; \overline{3}}\left(n_{1}, n_{2}\right)\left[K_{1} ; M_{1}, M_{2}\right] & =\int \frac{d^{4-2 \epsilon} p}{(2 \pi)^{4-2 \epsilon}} \frac{1}{\left(p^{2}-M_{1}^{2}\right)^{n_{1}}\left(\left(p-K_{1}\right)^{2}-M_{2}^{2}\right)^{n_{2}}} \\
I_{2 ; \overline{1}}\left(n_{1}, n_{2}\right)\left[K_{2} ; M_{2}, m_{1}\right] & =\int \frac{d^{4-2 \epsilon} p}{(2 \pi)^{4-2 \epsilon}} \frac{1}{\left(p^{2}-M_{2}^{2}\right)^{n_{1}}\left(\left(p-K_{2}\right)^{2}-m_{1}^{2}\right)^{n_{2}}} \\
I_{2 ; \overline{2}}\left(n_{2}, n_{1}\right)\left[K_{3} ; m_{1}, M_{1}\right] & =\int \frac{d^{4-2 \epsilon} p}{(2 \pi)^{4-2 \epsilon}} \frac{1}{\left(\left(p-K_{3}\right)^{2}-M_{1}^{2}\right)^{n_{1}}\left(p^{2}-m_{1}^{2}\right)^{n_{2}}}
\end{aligned}
$$

\footnotetext{
${ }^{7}$ The $\Delta\left[K_{1}, M_{1}, M_{2}\right]$ reflects the singularity of bubble as given in (2.5), while $\Delta_{3 ; m=0}$ corresponds the pure second-type Landau singularity of triangle.
} 
When we use the unitarity cut method to find the reduction coefficients, a given cut can only detect these coefficients of basis which have the corresponding cut. For each cut, it can detect the triangle coefficient and one bubble coefficient, while all tadpole coefficients can not be found by this way. For triangle, there are three different cuts, i.e, $K_{1}, K_{2}, K_{3}$. the coefficient of bubble $\mathcal{I}_{2 ; \overline{3}}$ can only be detected by the cut with momentum $K_{1}$ and similarly bubble coefficients of $\mathcal{I}_{2 ; \overline{1}}$ and $\mathcal{I}_{2 ; \overline{2}}$ by the cut $K_{2}$ and $K_{3}$ respectively. Thus using them all, we can get reduction coefficients of triangle and bubbles. Furthermore, there are overlaps of detected reduction coefficients between different cuts. For the triangle case, all three cuts can detect the same triangle coefficient, so we can use the overlap as the cross check.

\subsection{Recurrence relation}

Before going to explicit calculation, let us establish some recurrence relations like the one (2.27) and (2.29) in the previous section. To prepare for this task, we discuss some symmetric properties of the integral (3.1) first. By shifting the momentum $p$, we can rewrite (3.1) to different forms, for example, by shifting $p \rightarrow p+K_{1}$ we get

$$
\begin{aligned}
& I_{3}\left(n_{1}, n_{2}, n_{3}\right)\left[K_{1}, K_{2}, K_{3} ; M_{1}, M_{2}, m_{1}\right] \\
& \quad=\int \frac{d^{4-2 \epsilon} p^{4}}{(2 \pi)^{4-2 \epsilon}} \frac{1}{\left(\left(p+K_{1}\right)^{2}-M_{1}^{2}\right)^{n_{1}}\left(p^{2}-M_{2}^{2}\right)^{n_{2}}\left(\left(p-K_{2}\right)^{2}-m_{1}^{2}\right)^{n_{3}}} \\
& \quad=I_{3}\left(n_{2}, n_{3}, n_{1}\right)\left[K_{2}, K_{3}, K_{1} ; M_{2}, m_{1}, M_{1}\right]
\end{aligned}
$$

and similarly for the shifting $p \rightarrow p+K_{1}+K_{2}$. Above relations (3.14) by shifting can be summarized as the cyclic symmetry $Z_{3}$ of three ordered lists

$g_{3}:\left\{\left(n_{1}, n_{2}, n_{3}\right) ;\left(K_{1}, K_{2}, K_{3}\right) ;\left(M_{1}, M_{2}, m_{1}\right)\right\} \rightarrow\left\{\left(n_{2}, n_{3}, n_{1}\right) ;\left(K_{2}, K_{3}, K_{1}\right) ;\left(M_{2}, m_{1}, M_{1}\right)\right\}$

We can also consider the variable changing $p \rightarrow-p$ in (3.1) to $\operatorname{get}^{8}$

$$
\begin{aligned}
& I_{3}\left(n_{1}, n_{2}, n_{3}\right)\left[K_{1}, K_{2}, K_{3} ; M_{1}, M_{2}, m_{1}\right] \\
& \quad=\int \frac{d^{4-2 \epsilon} p}{(2 \pi)^{4-2 \epsilon}} \frac{1}{\left(p^{2}-M_{1}^{2}\right)^{n_{1}}\left(\left(p+K_{1}\right)^{2}-M_{2}^{2}\right)^{n_{2}}\left(\left(p-K_{3}\right)^{2}-m_{1}^{2}\right)^{n_{3}}} \\
& \quad=I_{3}\left(n_{1}, n_{3}, n_{2}\right)\left[K_{3}, K_{2}, K_{1} ; M_{1}, m_{1}, M_{2}\right]
\end{aligned}
$$

which can be summarized as the reflection symmetry $Z_{2}$ of three ordered lists

$g_{2}:\left\{\left(n_{1}, n_{2}, n_{3}\right) ;\left(K_{1}, K_{2}, K_{3}\right) ;\left(M_{1}, M_{2}, m_{1}\right)\right\} \rightarrow\left\{\left(n_{1}, n_{3}, n_{2}\right) ;\left(K_{3}, K_{2}, K_{1}\right) ;\left(M_{1}, m_{1}, M_{2}\right)\right\}$

When combining $g_{2}, g_{3}$ together, we generate the permutation group $S_{3}$. Using the symmetric property, we can connect the reduction of one integral to another integral. For example,

\footnotetext{
${ }^{8}$ When changing $p \rightarrow \widetilde{p}=-p$, the $\int_{-\infty}^{+\infty} d p \rightarrow \int_{+\infty}^{-\infty}-d \widetilde{p}=\int_{-\infty}^{+\infty} d \widetilde{p}$, i.e., the measure is invariant under the reflection.
} 
we know the expansion of $I_{3}(1,1, n)\left[K_{1}, K_{2}, K_{3} ; M_{1}, M_{2}, m_{1}\right]$ and we want to calculate the expansion of $I_{3}(1, n, 1)\left[K_{1}, K_{2}, K_{3} ; M_{1}, M_{2}, m_{1}\right]$. Using (3.14), we have

$$
\begin{aligned}
& I_{3}(1, n, 1)\left[K_{1}, K_{2}, K_{3} ; M_{1}, M_{2}, m_{1}\right]=g_{3}\left\{I_{3}(1,1, n)\left[K_{3}, K_{1}, K_{2} ; m_{1}, M_{1}, M_{2}\right]\right\} \\
& \quad=g_{3}\left\{c_{3 \rightarrow 3}\left(n_{1}, n_{2}, n_{3}\right) \mathcal{I}_{3}+\sum_{i=1}^{3} c_{3 \rightarrow 2 ; \bar{i}}\left(n_{1}, n_{2}, n_{3}\right) \mathcal{I}_{2 ; \bar{i}}+\sum_{i=1}^{3} c_{3 \rightarrow 1 ; i}\left(n_{1}, n_{2}, n_{3}\right) \mathcal{I}_{1 ; i}\right\} .
\end{aligned}
$$

Noticing that $\mathcal{I}_{3}\left[K_{1}, K_{2}, K_{3} ; M_{1}, M_{2}, m_{1}\right]=g_{3}\left\{\mathcal{I}_{3}\left[K_{3}, K_{1}, K_{2} ; m_{1}, M_{1}, M_{2}\right]\right\}$, we have

$$
c_{3 \rightarrow 3}(1, n, 1)\left[K_{1}, K_{2}, K_{3} ; M_{1}, M_{2}, m_{1}\right]=g_{3}\left\{c_{3 \rightarrow 3}(1,1, n)\left[K_{3}, K_{1}, K_{2} ; m_{1}, M_{1}, M_{2}\right]\right\} .
$$

Similarly, for bubble part we have

$$
\mathcal{I}_{2 ; \bar{i}}\left[K_{1}, K_{2}, K_{3} ; M_{1}, M_{2}, m_{1}\right]=g_{3}\left\{\mathcal{I}_{2 ; \bar{i}}\left[K_{3}, K_{1}, K_{2} ; m_{1}, M_{1}, M_{2}\right]\right\},
$$

thus

$$
c_{3 \rightarrow 2 ; i}(1, n, 1)\left[K_{1}, K_{2}, K_{3} ; M_{1}, M_{2}, m_{1}\right]=g_{3}\left\{c_{3 \rightarrow 2 ; i}(1,1, n)\left[K_{3}, K_{1}, K_{2} ; m_{1}, M_{1}, M_{2}\right]\right\} .
$$

One simple consequence of above symmetry property is that if we know the expansion of $I_{3}(1,1,2)$, we know also $I_{3}(1,2,1)$ and $I_{3}(2,1,1)$.

Now we show how to use $I_{3}(1,1,2), I_{3}(1,2,1)$ and $I_{3}(2,1,1)$ to get the expansion of general $I_{3}\left(n_{1}, n_{2}, n_{3}\right)$ using only differentiation. Let us start from $I_{3}\left(1,1, n_{3}\right)$ first. It is easy to see that

$$
\begin{aligned}
I_{3}\left(1,1, n_{3}\right)= & \frac{1}{\left(n_{3}-1\right)} \frac{d}{d\left(m_{1}^{2}\right)} I_{3}\left(1,1, n_{3}-1\right) \\
= & \frac{1}{\left(n_{3}-1\right)} \frac{d}{d\left(m_{1}^{2}\right)}\left\{c_{3 \rightarrow 3}\left(1,1, n_{3}-1\right) \mathcal{I}_{3}+\sum_{i=1}^{3} c_{3 \rightarrow 2 ; \bar{i}}\left(1,1, n_{3}-1\right) \mathcal{I}_{2 ; \bar{i}}+\ldots\right\} \\
= & \frac{1}{\left(n_{3}-1\right)} \frac{d c_{3 \rightarrow 3}\left(1,1, n_{3}-1\right)}{d\left(m_{1}^{2}\right)} \mathcal{I}_{3}+\frac{c_{3 \rightarrow 3}\left(1,1, n_{3}-1\right)}{\left(n_{3}-1\right)} I_{3}(1,1,2) \\
& +\sum_{i=1}^{3} \frac{d c_{3 \rightarrow 2 ; \bar{i}}\left(1,1, n_{3}-1\right)}{\left(n_{3}-1\right) d\left(m_{1}^{2}\right)} \mathcal{I}_{2 ; \bar{i}}+\frac{c_{3 \rightarrow 2 ; \overline{1}}\left(1,1, n_{3}-1\right)}{\left(n_{3}-1\right)} I_{2 ; \overline{1}}(1,2) \\
& +\frac{c_{3 \rightarrow 2 ; \overline{2}}\left(1,1, n_{3}-1\right)}{\left(n_{3}-1\right)} I_{2 ; \overline{2}}(2,1)+\ldots
\end{aligned}
$$

where in the last line, since the $\mathcal{I}_{2 ; \overline{3}}$ does not depend on $m_{1}$, the action of $\frac{d}{d m_{1}^{2}}$ is zero (see (3.10), (3.11) and (3.12)). Now, using the expansion of $I_{3}(1,1,2), I_{2}(1,2)$ and $I_{2}(2,1)$, we get immediately the recurrence relation

$$
\begin{aligned}
c_{3}\left(1,1, n_{3}\right)= & \frac{1}{\left(n_{3}-1\right)} \frac{d c_{3 \rightarrow 3}\left(1,1, n_{3}-1\right)}{d\left(m_{1}^{2}\right)}+\frac{c_{3 \rightarrow 3}\left(1,1, n_{3}-1\right)}{\left(n_{3}-1\right)} c_{3 \rightarrow 3}(1,1,2) \\
c_{3 \rightarrow 2 ; \overline{1}}\left(1,1, n_{3}\right)= & \frac{c_{3 \rightarrow 3}\left(1,1, n_{3}-1\right)}{\left(n_{3}-1\right)} c_{3 \rightarrow 2 ; \overline{1}}(1,1,2)+\frac{1}{\left(n_{3}-1\right)} \frac{d c_{3 \rightarrow 2 ; \overline{1}}\left(1,1, n_{3}-1\right)}{d\left(m_{1}^{2}\right)} \\
& +\frac{c_{3 \rightarrow 2 ; \overline{1}}\left(1,1, n_{3}-1\right)}{\left(n_{3}-1\right)} c_{2 \rightarrow 2 ; \overline{1}}(1,2)
\end{aligned}
$$




$$
\begin{aligned}
c_{3 \rightarrow 2 ; \overline{2}}\left(1,1, n_{3}\right)= & \frac{c_{3 \rightarrow 3}\left(1,1, n_{3}-1\right)}{\left(n_{3}-1\right)} c_{3 \rightarrow 2 ; \overline{2}}(1,1,2)+\frac{1}{\left(n_{3}-1\right)} \frac{d c_{3 \rightarrow 2 ; \overline{2}}\left(1,1, n_{3}-1\right)}{d\left(m_{1}^{2}\right)} \\
& +\frac{c_{3 \rightarrow 2 ; \overline{2}}\left(1,1, n_{3}-1\right)}{(n-1)} c_{2 \rightarrow 2 ; \overline{2}}(2,1) \\
c_{3 \rightarrow 2 ; \overline{3}}\left(1,1, n_{3}\right)= & \frac{c_{3 \rightarrow 3}\left(1,1, n_{3}-1\right)}{\left(n_{3}-1\right)} c_{3 \rightarrow 2 ; \overline{3}}(1,1,2)+\frac{1}{\left(n_{3}-1\right)} \frac{d c_{3 \rightarrow 2 ; \overline{3}}\left(1,1, n_{3}-1\right)}{d\left(m_{1}^{2}\right)}
\end{aligned}
$$

Knowing (3.22), it is easy to use (3.15) and (3.17) to get reduction coefficients for $I_{3}(1, n, 1)$ and $I_{3}(n, 1,1)$ as shown in (3.18). One point we want to emphasize is that since $g_{2}, g_{3}$ acts on $K, M$ also, the kinematic dependence of $K, M$ in (3.22) should be carefully identified although for simplicity we have not written them down explicitly.

Knowing the reduction of $I_{3}\left(1,1, n_{3}\right)$, now we consider the case $I_{3}\left(1, n_{2}, n_{3}\right)$. Similarly to (3.21), we have

$$
I_{3}\left(1, n_{2}, n_{3}\right)=\frac{1}{\left(n_{2}-1\right)} \frac{\partial}{\partial M_{2}^{2}}\left\{c_{3 \rightarrow 3}\left(1, n_{2}-1, n_{3}\right) I_{3}+\sum_{i=1}^{3} c_{3 \rightarrow 2 ; \bar{i}}\left(1, n_{2}-1, n_{3}\right) I_{2 ; \bar{i}}+\cdots\right\}
$$

thus we can get the recurrence relation for reduction coefficients

$$
\begin{aligned}
c_{3 \rightarrow 3}\left(1, n_{2}, n_{3}\right)= & \frac{1}{n_{2}-1}\left(\frac{\partial c_{3 \rightarrow 3}\left(1, n_{2}-1, n_{3}\right)}{\partial M_{2}^{2}}+c_{3 \rightarrow 3}\left(1, n_{2}-1, n_{3}\right) c_{3 \rightarrow 3}(1,2,1)\right) \\
c_{3 \rightarrow 2 ; \overline{1}}\left(1, n_{2}, n_{3}\right)= & \frac{1}{n_{2}-1}\left(c_{3 \rightarrow 3}\left(1, n_{2}-1, n_{3}\right) c_{3 \rightarrow 2 ; \overline{1}}(1,2,1)+\frac{\partial c_{3 \rightarrow 2 ; \overline{1}}\left(1, n_{2}-1, n_{3}\right)}{\partial M_{2}^{2}}\right. \\
& \left.+c_{3 \rightarrow 2 ; \overline{1}}\left(1, n_{2}-1, n_{3}\right) c_{2 \rightarrow 2, \overline{1}}(2,1)\right) \\
c_{3 \rightarrow 2 ; \overline{2}}\left(1, n_{2}, n_{3}\right)= & \frac{1}{n_{2}-1}\left(c_{3 \rightarrow 3}\left(1, n_{2}-1, n_{3}\right) c_{3 \rightarrow 2 ; \overline{2}}(1,2,1)+\frac{\partial c_{3 \rightarrow 2 ; \overline{2}}\left(1, n_{2}-1, n_{3}\right)}{\partial M_{2}^{2}}\right) \\
c_{3 \rightarrow 2 ; \overline{3}}\left(1, n_{2}, n_{3}\right)= & \frac{1}{n_{2}-1}\left(c_{3 \rightarrow 3}\left(1, n_{2}-1, n_{3}\right) c_{3 \rightarrow 2 ; \overline{3}}(1,2,1)+\frac{\partial c_{3 \rightarrow 2 ; \overline{3}}\left(1, n_{2}-1, n_{3}\right)}{\partial M_{2}^{2}}\right. \\
& \left.+c_{3 \rightarrow 2 ; \overline{3}}\left(1, n_{2}-1, n_{3}\right) c_{2 \rightarrow 2}(1,2)\right)
\end{aligned}
$$

So if we have known the expansion coefficients of $I_{3}\left(1, n_{2}-1, n_{3}\right)$ to the basis $I_{3}$ and $I_{2}$, we can derive the expansion of $I_{3}\left(1, n_{2}, n_{3}\right)$.

Finally, for $I_{3}\left(n_{1}, n_{2}, n_{3}\right)$ with $n_{1}>1$, similar action lead to the recurrence relation

$$
\begin{aligned}
c_{3 \rightarrow 3}\left(n_{1}, n_{2}, n_{3}\right)= & \frac{1}{n_{1}-1}\left(\frac{\partial c_{3 \rightarrow 3}\left(n_{1}-1, n_{2}, n_{3}\right)}{\partial M_{1}^{2}}+c_{3 \rightarrow 3}\left(n_{1}-1, n_{2}, n_{3}\right) c_{3 \rightarrow 3}(2,1,1)\right) \\
c_{3 \rightarrow 2 ; \overline{1}}= & \frac{1}{n_{1}-1}\left(c_{3 \rightarrow 3}\left(n_{1}-1, n_{2}, n_{3}\right) c_{3 \rightarrow 2 ; \overline{1}}(2,1,1)+\frac{\partial c_{3 \rightarrow 2 ; \overline{1}}\left(n_{1}-1, n_{2}, n_{3}\right)}{\partial M_{1}^{2}}\right) \\
c_{3 \rightarrow 2 ; \overline{2}}= & \frac{1}{n_{1}-1}\left(c_{3 \rightarrow 3}\left(n_{1}-1, n_{1}, n_{3}\right) c_{3 \rightarrow 2 ; \overline{2}}(2,1,1)+\frac{\partial c_{3 \rightarrow 2 ; \overline{2}}\left(n_{1}-1, n_{2}, n_{3}\right)}{\partial M_{1}^{2}}\right. \\
& \left.+c_{3 \rightarrow 2 ; \overline{2}}\left(n_{1}-1, n_{2}, n_{3}\right) c_{2 \rightarrow 2 ; \overline{2}}(2,1)\right)
\end{aligned}
$$




$$
\begin{aligned}
c_{2 ; \overline{3}}= & \frac{1}{n_{1}-1}\left(c_{3}\left(n_{1}-1, n_{2}, n_{3}\right) c_{2 ; \overline{3}}(2,1,1)+\frac{\partial c_{2 ; \overline{3}}\left(n_{1}-1, n_{2}, n_{3}\right)}{\partial M_{1}^{2}}\right. \\
& \left.+c_{2 ; \overline{3}}\left(n_{1}-1, n_{2}, n_{3}\right) c_{2,1}\left[K_{1}, M_{1}, M_{2}\right]\right)
\end{aligned}
$$

\subsection{Triangle $(1,1,2)$}

From the recurrence relation in previous subsection, we see that all computations have been reduced to the reduction of $I_{3}(1,1,2)$. Although there are other methods to reduce $I_{3}(1,1,2)$ such as the IBP method. In this part, we show how to use unitarity cut method to fulfill the task. Using our idea, we can write

$$
I_{3}(1,1,2)=\frac{\partial}{\partial m_{1}^{2}} I_{3}(1,1,1) .
$$

To get all reduction coefficients, we need to calculate all three cuts. However, different cuts can be related using the symmetry discussed in previous subsection, thus we will focus on only one cut.

\subsubsection{Cut $K_{1}$}

For this cut, the r.h.s. is given by

$$
\mathcal{C}_{K_{1}}\left(I_{3}(1,1,2)\right)=\frac{\partial}{\partial m_{1}^{2}} \mathcal{C}\left(I_{3}\right)=-\left(\frac{4 K_{1}^{2}}{\Delta\left[K_{1}, M_{1}, M_{2}\right]}\right)^{\epsilon} \frac{1}{\sqrt{\Delta_{3 ; m=0}}} \frac{\partial}{\partial m_{1}^{2}} \operatorname{Tri}^{(0)}(Z)
$$

where we have used the fact that only $Z$ contains the $m_{1}$. Carrying out the derivative, we get

$$
\begin{aligned}
\frac{\partial}{\partial m_{1}^{2}} \operatorname{Tri}^{(0)}(Z) & =\frac{\partial Z}{\partial m_{1}^{2}} \frac{\partial}{\partial Z} \operatorname{Tri}^{(0)}(Z), \quad \frac{\partial Z}{\partial m_{1}^{2}}=\frac{2 K_{1}^{2}}{\sqrt{\Delta_{3 ; m=0} \Delta\left[K_{1}, M_{1}, M_{2}\right]}} \\
\frac{\partial}{\partial Z} \operatorname{Tri}^{(0)}(Z) & =\int_{0}^{1} d u u^{-1-\epsilon}\left[\frac{1}{Z+\sqrt{1-u}}-\frac{1}{Z-\sqrt{1-u}}\right] \\
& =2 \int_{0}^{1} d u u^{-1-\epsilon} \frac{1-u}{\sqrt{1-u}\left(1-u-Z^{2}\right)}
\end{aligned}
$$

Among two terms of $\frac{\partial}{\partial Z} \operatorname{Tr}^{(0)}(Z)$, by comparing with (3.3), the second term is

$$
2 \int_{0}^{1} d u u^{-1-\epsilon} \frac{-u}{\sqrt{1-u}\left(1-u-Z^{2}\right)}=-\frac{2 \epsilon}{Z} \operatorname{Tri}^{(0)}(Z)
$$

For the first term, noting that

$$
\int_{0}^{1} d u u^{-1-\epsilon} \frac{1}{\sqrt{1-u}}=\left(1-Z^{2}\right) \int_{0}^{1} d u u^{-1-\epsilon} \frac{1}{\sqrt{1-u}\left(1-u-Z^{2}\right)}-\int_{0}^{1} d u u^{-\epsilon} \frac{1}{\sqrt{1-u}\left(1-u-Z^{2}\right)}
$$

we have

$$
\frac{1}{1-Z^{2}} \frac{\epsilon}{Z} \operatorname{Tri}^{(0)}(Z)+\frac{1-2 \epsilon}{1-Z^{2}} B u b^{(0)}
$$


Putting all together, we get

$$
\frac{\partial}{\partial Z} \operatorname{Tr}^{(0)}(Z)=\frac{2(1-2 \epsilon)}{1-Z^{2}} B u b^{(0)}+\frac{2 Z \epsilon}{1-Z^{2}} \operatorname{Tri}^{(0)}(Z)
$$

By identifying

$$
\frac{\partial}{\partial m_{1}^{2}} \operatorname{CutI}_{3}(1,1,1)=c_{3 \rightarrow 3 ; K_{1}}(1,1,2) \operatorname{CutI}_{3}(1,1,1)+c_{3 \rightarrow 2 ; \overline{3} ; K_{1}}(1,1,2) \operatorname{CutI}_{2}(1,1)
$$

we get the reduction coefficients as

$$
\begin{gathered}
c_{3 \rightarrow 3 ; K_{1}}(1,1,2)=\frac{4 K_{1}^{2}}{\sqrt{\Delta_{3 ; m=0} \Delta\left[K_{1}, M_{1}, M_{2}\right]}} \frac{Z \epsilon}{1-Z^{2}} \\
c_{3 \rightarrow 2 ; \overline{3} ; K_{1}}(1,1,2)=-\frac{4 K_{1}^{2}}{\Delta\left[K_{1}, M_{1}, M_{2}\right] \Delta_{3 ; m=0}} \frac{1-2 \epsilon}{1-Z^{2}}
\end{gathered}
$$

where for simplicity we have not expanded $Z$ further (see (3.4)). We want to remark that the coefficients $c_{3 \rightarrow 3 ; K_{1}}$ and $c_{3 \rightarrow 2 ; \overline{3} ; K_{1}}$ are nothing, but the $c_{3 \rightarrow 3}$ and $c_{3 \rightarrow 2 ; \overline{3}}$ is the reduction

$$
I_{3}(1,1,2)=c_{3 \rightarrow 3}(1,1,2) I_{3}+c_{3 \rightarrow 2 ; \overline{3}}(1,1,2) I_{3 ; \overline{3}}+\cdots \cdots
$$

The reason that we have added subscript $K_{1}$ in (3.34) and (3.35) is to emphasize that these two expressions are calculated using the cut $K_{1}$.

\subsubsection{Cut $K_{2}$}

No we consider the cut $K_{2}$. The use the cut result (3.2), we need to rewrite the form (3.1) into the standard form for the cut $K_{2}$ by shifting the integral momentum $p \rightarrow p+K_{1}$, thus it becomes

$$
\begin{aligned}
I_{3}( & \left.n_{1}, n_{2}, n_{3}\right)\left[K_{1}, K_{2}, K_{3} ; M_{1}, M_{2}, m_{1}\right] \\
& =\int \frac{d^{4-2 \epsilon} p}{(2 \pi)^{4-2 \epsilon}} \frac{1}{\left(\left(p+K_{1}\right)^{2}-M_{1}^{2}\right)^{n_{1}}\left(p^{2}-M_{2}^{2}\right)^{n_{2}}\left(\left(p-K_{2}\right)^{2}-m_{1}^{2}\right)^{n_{3}}} \\
& =I_{3}\left(n_{2}, n_{3}, n_{1}\right)\left[K_{2}, K_{3}, K_{1} ; M_{2}, m_{1}, M_{1}\right] \\
& =g_{3}\left\{I_{3}\left(n_{1}, n_{2}, n_{3}\right)\left[K_{1}, K_{2}, K_{3} ; M_{1}, M_{2}, m_{1}\right]\right\}
\end{aligned}
$$

For the case $n_{3}=2, n_{1}=n_{2}=1$

$$
\begin{aligned}
I_{3}(1,1,2)\left[K_{1}, K_{2}, K_{3} ; M_{1}, M_{2}, m_{1}\right] & =\frac{\partial}{\partial m_{1}^{2}} I_{3}(1,1,1)\left[K_{1}, K_{2}, K_{3} ; M_{1}, M_{2}, m_{1}\right] \\
& =\frac{\partial}{\partial m_{1}^{2}} I_{3}(1,1,1)\left[K_{2}, K_{3}, K_{1} ; M_{2}, m_{1}, M_{1}\right] \\
& =g_{3}\left\{\frac{\partial}{\partial M_{2}^{2}} I_{3}(1,1,1)\left[K_{1}, K_{2}, K_{3} ; M_{1}, M_{2}, m_{1}\right]\right\}
\end{aligned}
$$

Thus we have reduced the problem to the cut $K_{1}$, but with different action $\frac{\partial}{\partial M_{2}^{2}}$. 
For the action of $\frac{\partial}{\partial M_{2}^{2}}$, there are two terms containing the parameter $M_{2}$ in the Cut $_{K_{1}}\left(I_{3}(1,1,2)\right)$.

$$
\begin{aligned}
\frac{\partial}{\partial M_{2}^{2}} \operatorname{Cut}_{K_{1}}\left(I_{3}(1,1,2)\right)= & -\frac{1}{\sqrt{\Delta_{3 ; m=0}}}\left(\frac{\partial}{\partial M_{2}^{2}}\left(\frac{\Delta}{4 K_{1}^{2}}\right)^{-\epsilon}\right) \operatorname{Tr} i^{(0)}(Z) \\
& +\left(-\frac{1}{\sqrt{\Delta_{3 ; m=0}}}\right)\left(\frac{\Delta}{4 K_{1}^{2}}\right)^{-\epsilon} \frac{\partial}{\partial M_{2}^{2}} \operatorname{Tri}^{(0)}(Z)
\end{aligned}
$$

where the $\Delta$ means $\Delta\left[K_{1}, M_{1}, M_{2}\right]$. The first term in (3.39) is trivial, while the derivative part in the second term is just $\frac{\partial Z}{\partial M_{2}^{2}} \frac{\partial}{\partial Z} \operatorname{Tr} i^{(0)}(Z)$, where $\frac{\partial}{\partial Z} \operatorname{Tr} i^{(0)}(Z)$ has been given in (3.32). Put them altogether, we get

$$
\frac{\partial}{\partial M_{2}^{2}} C_{K_{1}}\left(I_{3}\right)=\widetilde{c}_{3} C\left(I_{3}\right)+\widetilde{c}_{2} C\left(I_{2}\right)
$$

with

$$
\tilde{c}_{3}=\Delta^{\epsilon} \frac{\partial}{\partial M_{2}^{2}} \Delta^{-\epsilon}+\frac{\partial Z}{\partial M_{2}^{2}} \frac{2 Z \epsilon}{1-Z^{2}}, \quad \tilde{c}_{2}=-\frac{1}{\sqrt{\Delta_{3 ; m=0}}} \frac{K_{1}^{2}}{\sqrt{\Delta}} \frac{\partial Z}{\partial M_{2}^{2}} \frac{2(1-2 \epsilon)}{1-Z^{2}}
$$

Of course, the $\tilde{c}_{2}$ and $\tilde{c}_{3}$ in (3.41) are not the final result, since we have to do the permutation $g_{3}$ to get $c_{3 \rightarrow 3}(1,1,2)=g_{3}\left(\widetilde{c}_{2}\right)$ and $c_{3 \rightarrow 2 ; \overline{1}}(1,1,2)=g_{3}\left(\widetilde{c}_{2}\right)$. One can find that the $g_{3}\left(\widetilde{c}_{2}\right)$ is equal to $c_{3 \rightarrow 3}(1,1,2)$ given in $(3.34)$, thus we have passed the first consistent check.

\subsubsection{Cut $K_{3}$}

Now we consider the cut $K_{3}$. Again we need to rewrite the form (3.1) into the standard form for the cut $K_{3}$ by shifting the integral momentum $p \rightarrow p+K_{1}+K_{2}$. With similar argument we will have

$$
\begin{aligned}
I_{3}(1,1,2)\left[K_{1}, K_{2}, K_{3} ; M_{1}, M_{2}, m_{1}\right] & =\frac{\partial}{\partial m_{1}^{2}} I_{3}(1,1,1)\left[K_{3}, K_{1}, K_{2} ; m_{1}, M_{1}, M_{2}\right] \\
& =g_{3}^{-1}\left\{\frac{\partial}{\partial M_{1}^{2}} I_{3}(1,1,2)\left[K_{1}, K_{2}, K_{3} ; M_{1}, M_{2}, m_{1}\right]\right\}
\end{aligned}
$$

thus the cut $K_{3}$ has been reduced to the cut $K_{1}$ with the action $\frac{\partial}{\partial M_{1}^{2}}$. The calculation is similar to the previous subsection. After simplification, we have

$$
\frac{\partial}{\partial M_{1}^{2}} C_{K_{1}}\left(I_{3}\right)=\widehat{c}_{3} C_{K_{1}}\left(I_{3}\right)+\widehat{c}_{2} C_{K_{1}}\left(I_{2}\right)
$$

with the coefficients

$$
\begin{aligned}
& \widehat{c}_{3}=-\epsilon \Delta^{-1} \frac{\partial \Delta}{\partial M_{1}^{2}}+\frac{\partial Z}{\partial M_{1}^{2}} \frac{2 Z \epsilon}{1-Z^{2}} \\
& \widehat{c}_{2}=\left(-\frac{1}{\sqrt{\Delta_{3 ; m=0}}}\right) \frac{K_{1}^{2}}{\sqrt{\Delta}} \frac{\partial Z}{\partial M_{1}^{2}} \frac{2(1-2 \epsilon)}{1-Z^{2}}
\end{aligned}
$$

After doing the permutation $g_{3}^{-1}$ on the coefficients above, we get the $c_{3 \rightarrow 3}$ and $c_{3 \rightarrow 2 ; \overline{2}}$ in this cut. Again, one can check the $c_{3 \rightarrow 3}$ is the same with $c_{3 \rightarrow 3 ; K_{1}}$ in (3.34). 
For the case $I_{3}(1,1,2)$ there is another consistent check we can do. Noticing that when we do following changing variable $p \rightarrow-p+K_{1}$, the (3.1) becomes to

$$
I_{3}\left(n_{1}, n_{2}, n_{3}\right)[1]=\int \frac{d^{4-2 \epsilon} p}{(2 \pi)^{4-2 \epsilon}} \frac{1}{\left(\left(p-K_{1}\right)^{2}-M_{1}^{2}\right)^{n_{1}}\left(p^{2}-M_{2}^{2}\right)^{n_{2}}\left(\left(p+K_{2}\right)-m_{1}^{2}\right)^{n_{3}}}
$$

Thus for $\left(n_{1}, n_{2}, n_{3}\right)=(1,1,2)$, there is the symmetry among the reduction coefficients, i.e., under the permutation $K_{2} \longleftrightarrow K_{3}, M_{1} \longleftrightarrow M_{2}$, we should have $c_{3 \rightarrow 3}$ invariant and $c_{3 \rightarrow 2 ; \overline{2}} \leftrightarrow c_{3 \rightarrow 2 ; \overline{1}}$. This can be easily checked by MATHEMATICA for our results.

\subsubsection{A short summary}

In the subsection, we have reduced the $I_{3}(1,1,2)$ to triangle $I_{3}$ and bubble $I_{2}$. After taking three different cuts, we get the all needed triangle and bubble coefficients of the reduction of $I_{3}(1,1,2)$

$$
I_{3}(1,1,2)=c_{3 \rightarrow 3}(1,1,2) I_{3}+\sum_{i=1}^{3} c_{3 \rightarrow 2 ; \bar{i}}(1,1,2) I_{2 ; \bar{i}}+\cdots
$$

where the $\cdots$ represents the tadpoles neglected in the whole paper. The coefficients in (3.46) are

$$
\begin{aligned}
c_{3 \rightarrow 3}(1,1,2) & =\frac{4 K_{1}^{2}}{\sqrt{\Delta_{3 ; m=0} \Delta\left[K_{1}, M_{1}, M_{2}\right]}} \frac{Z \epsilon}{1-Z^{2}} \\
c_{3 \rightarrow 2 ; \overline{3}}(1,1,2) & =-\frac{4 K_{1}^{4}}{\Delta\left[K_{1}, M_{1}, M_{2}\right] \Delta_{3 ; m=0}} \frac{1-2 \epsilon}{1-Z^{2}} \\
c_{3 \rightarrow 2 ; \overline{1}}(1,1,2) & =\hat{g}_{3}\left(-\frac{1}{\sqrt{\Delta_{3 ; m=0}}} \frac{K_{1}^{2}}{\sqrt{\Delta\left[K_{1}, M_{1}, M_{2}\right]}} \frac{\partial Z}{\partial M_{2}^{2}} \frac{2(1-2 \epsilon)}{1-Z^{2}}\right) \\
c_{3 \rightarrow 2 ; \overline{2}}(1,1,2) & =\hat{g}_{3}^{-1}\left(-\frac{1}{\sqrt{\Delta_{3 ; m=0}}} \frac{K_{1}^{2}}{\sqrt{\Delta\left[K_{1}, M_{1}, M_{2}\right]}} \frac{\partial Z}{\partial M_{1}^{2}} \frac{2(1-2 \epsilon)}{1-Z^{2}}\right)
\end{aligned}
$$

The result is confirmed with IBP method using LiteRed [23]. We have also carried out the reduction of $I_{3}(1,2,1), I_{3}(2,1,1)$ and $I_{3}(1,2,2)$ using the method laid out in the paper and found perfect match with the IBP method.

\section{Box}

For the box topology, we define

$$
\begin{aligned}
& I_{4}\left(n_{1}, n_{2}, n_{3}, n_{4}\right) \\
& =\int \frac{d^{4-2 \epsilon} p}{(2 \pi)^{4-2 \epsilon}} \frac{1}{\left(p^{2}-M_{1}^{2}\right)^{n_{1}}\left(\left(p-K_{1}\right)^{2}-M_{2}^{2}\right)^{n_{2}}\left(\left(p-K_{1}-K_{2}\right)^{2}-m_{1}^{2}\right)^{n_{3}}\left(\left(p+K_{4}\right)^{2}-m_{2}^{2}\right)^{n_{4}}} \\
& I_{4}\left(n_{1}, n_{2}, n_{3}, n_{4}\right) \\
& =\int \frac{d^{4-2 \epsilon} p}{(2 \pi)^{4-2 \epsilon}} \frac{1}{\left(p^{2}-M_{1}^{2}\right)^{n_{1}}\left((p-K)^{2}-M_{2}^{2}\right)^{n_{2}}\left(\left(p-P_{1}\right)^{2}-m_{1}^{2}\right)^{n_{3}}\left(\left(p-P_{2}\right)^{2}-m_{2}^{2}\right)^{n_{4}}}
\end{aligned}
$$

or

for later convenience, where we have labeled the cut momentum as $K$ and the masses of two cut propagators as $M_{1}$ and $M_{2}$ respectively. For the other two momenta we denote 
them as $P_{1}$ and $P_{2}$ respectively. For example, if we choose $K_{1}$ as the cut momentum, we will have $P_{1}=K_{1}+K_{2}$ and $P_{2}=-K_{4}$, but if the cut momentum is $K_{1}+K_{2}$, we will have $P_{1}=K_{1}, P_{2}=K_{1}+K_{2}+K_{3}$.

The special case with $n_{i}=1, i=1,2,3,4$ gives the scalar basis, which we will denote as $I_{4}$. The unitarity cut of $I_{4}$ with the cut momentum $K$ has been given by [9]

$$
C\left(I_{4}\right)=\left(\frac{\Delta}{4 K^{2}}\right)^{-\epsilon} \frac{b}{2 K^{2}} \int_{0}^{1} d u u^{-1-\epsilon} \frac{1}{\sqrt{B-A u}} \ln \left(\frac{D-C u+\sqrt{1-u} \sqrt{B-A u}}{D-C u-\sqrt{1-u} \sqrt{B-A u}}\right)
$$

with the parameters $\Delta$ in $(2.5)$ and $^{9}$

$$
\begin{array}{rlrl}
A & =-\frac{b^{4}}{K^{2}} \operatorname{det}\left(\begin{array}{ccc}
P_{1}^{2} & P_{1} \cdot P_{2} & P_{1} \cdot K \\
P_{1} \cdot P_{2} & P_{2}^{2} & P_{2} \cdot K \\
P_{1} \cdot K & P_{2} \cdot K & K^{2}
\end{array}\right), & B=-\operatorname{det}\left(\begin{array}{cc}
R_{1}^{2} & R_{1} \cdot R_{2} \\
R_{1} \cdot R_{2} & R_{2}^{2}
\end{array}\right) \\
C=\frac{b^{2}}{K^{2}} \operatorname{det}\left(\begin{array}{cc}
P_{1} \cdot P_{2} & P_{1} \cdot K \\
P_{2} \cdot K & K^{2}
\end{array}\right), & D=R_{1} \cdot R_{2}
\end{array}
$$

where

$$
b=\frac{\sqrt{\Delta\left[K, M_{1}, M_{2}\right]}}{K^{2}}, \quad a_{i}=\frac{P_{i}^{2}+M_{1}^{2}-m_{1}^{2}}{K^{2}}, \quad R_{i}=-b P_{i}+\left(a_{i}-\frac{P_{i} \cdot K}{K^{2}}(a-b)\right) K
$$

With the definition

$$
B o x^{(n)}=\int_{0}^{1} d u u^{-1-\epsilon} \frac{u^{n}}{\sqrt{B-A u}} \ln \left(\frac{D-C u+\sqrt{1-u} \sqrt{B-A u}}{D-C u-\sqrt{1-u} \sqrt{B-A u}}\right)
$$

we can write $C\left(I_{4}\right)$ as

$$
C\left(I_{4}\right)=\left(\frac{\Delta}{4 K^{2}}\right)^{-\epsilon} \frac{b}{2 K^{2}} B o x^{(0)}
$$

and there is also a recurrence relation of $B o x^{(n)}$, which is given by $[8,9]$

$$
B o x^{(n)}=\frac{n-1-\epsilon}{n-\frac{1}{2}-\epsilon} \frac{B}{A} B o x^{(n-1)}-\frac{n-1-\epsilon}{n-\frac{1}{2}-\epsilon} \frac{C_{Z_{1}}}{A Z_{1}} \operatorname{Tri}^{(n-1)}\left(Z_{1}\right)-\frac{n-1-\epsilon}{n-\frac{1}{2}-\epsilon} \frac{C_{Z_{2}}}{A Z_{2}} \operatorname{Tri}^{(n-1)}\left(Z_{2}\right)
$$

with the parameter

$$
C_{Z_{i}}=D+\left(Z_{i}^{2}-1\right) C
$$

To be calar, we list six possible cuts of a box, with $K_{1}, K_{2}, K_{3}, K_{4}$ in clockwise ordering. And there will be two cut triangles related to each given cut momentum $K$ of box:

\begin{tabular}{|c|c|c|c|c|}
\hline Box Cut $K$ & $P_{1}$ & $P_{2}$ & Triangle One's $\left(K_{1}, K_{3}\right)$ & Triangle Two's $\left(K_{1}, K_{3}\right)$ \\
\hline$K_{1}$ & $K_{12}$ & $-K_{4}$ & $\left(K_{1}, K_{34}\right)$ & $\left(K_{1}, K_{4}\right)$ \\
\hline$K_{2}$ & $K_{23}$ & $-K_{1}$ & $\left(K_{2}, K_{41}\right)$ & $\left(K_{2}, K_{1}\right)$ \\
\hline$K_{3}$ & $K_{34}$ & $-K_{2}$ & $\left(K_{3}, K_{12}\right)$ & $\left(K_{3}, K_{2}\right)$ \\
\hline$K_{4}$ & $K_{41}$ & $-K_{3}$ & $\left(K_{4}, K_{23}\right)$ & $\left(K_{4}, K_{3}\right)$ \\
\hline$K_{12}$ & $K_{1}$ & $-K_{4}$ & $\left(K_{34}, K_{2}\right)$ & $\left(K_{12}, K_{4}\right)$ \\
\hline$K_{23}$ & $K_{2}$ & $-K_{1}$ & $\left(K_{41}, K_{3}\right)$ & $\left(K_{23}, K_{1}\right)$ \\
\hline
\end{tabular}

\footnotetext{
${ }^{9}$ The $A$ corresponds to the second-type singularity of box.
} 
Symmetry analysis. Similar to the case of triangle, there are some symmetries of box by momentum shifting and reflection. Let us define two generators

$$
\begin{aligned}
\hat{g}_{4}: & \left\{\left(\left(n_{1}, n_{2}, n_{3}, n_{4}\right) ;\left(K_{1}, K_{2}, K_{3}, K_{4}\right) ;\left(M_{1}, M_{2}, m_{1}, m_{2}\right)\right\}\right. \\
& \rightarrow\left\{\left(n_{2}, n_{3}, n_{4}, n_{1}\right) ;\left(K_{2}, K_{3}, K_{4}, K_{1}\right) ;\left(M_{2}, m_{1}, m_{2}, M_{1}\right)\right\}
\end{aligned}
$$

and

$$
\begin{aligned}
\hat{g}_{2}: & \left\{\left(\left(n_{1}, n_{2}, n_{3}, n_{4}\right) ;\left(K_{1}, K_{2}, K_{3}, K_{4}\right) ;\left(M_{1}, M_{2}, m_{1}, m_{2}\right)\right\}\right. \\
& \rightarrow\left\{\left(n_{1}, n_{4}, n_{3}, n_{2}\right) ;\left(K_{4}, K_{3}, K_{2}, K_{1}\right) ;\left(M_{1}, m_{2}, m_{1}, M_{2}\right)\right\}
\end{aligned}
$$

Using them we get the dihedral group $D_{4}$ and following relations

$$
\begin{aligned}
& I_{4}\left(n_{2}, n_{3}, n_{4}, n_{1}\right)\left[K_{2}, K_{3}, K_{4}, K_{1} ; M_{2}, m_{1}, m_{2}, M_{1}\right] \\
& \quad=\widehat{g}_{4} I_{4}\left(n_{1}, n_{2}, n_{3}, n_{4}\right)\left[K_{1}, K_{2}, K_{3}, K_{4} ; M_{1}, M_{2}, m_{1}, m_{2}\right] \\
& \quad \quad=\widehat{g}_{4}^{2} I_{4}\left(n_{1}, n_{2}, n_{3}, n_{4}\right)\left[K_{1}, K_{2}, K_{3}, K_{4} ; M_{1}, M_{2}, m_{1}, m_{2}\right] \\
& \quad \quad=\widehat{g}_{4}^{3} I_{4}\left(n_{1}, n_{2}, n_{3}, n_{4}\right)\left[K_{1}, K_{2}, K_{3}, K_{4} ; M_{1}, M_{2}, m_{1}, m_{2}\right]
\end{aligned}
$$

and

$$
\begin{aligned}
& I_{4}\left(n_{1}, n_{4}, n_{3}, n_{2}\right)\left[K_{4}, K_{3}, K_{2}, K_{1} ; M_{1}, m_{2}, m_{1}, M_{2}\right] \\
& =\widehat{g}_{2} I_{4}\left(n_{1}, n_{2}, n_{3}, n_{4}\right)\left[K_{1}, K_{2}, K_{3}, K_{4} ; M_{1}, M_{2}, m_{1}, m_{2}\right] \\
& I_{4}\left(n_{4}, n_{3}, n_{2}, n_{1}\right)\left[K_{3}, K_{2}, K_{1}, K_{4} ; m_{2}, m_{1}, M_{2}, M_{1}\right] \\
& =\widehat{g}_{4} \widehat{g}_{2} I_{4}\left(n_{1}, n_{2}, n_{3}, n_{4}\right)\left[K_{1}, K_{2}, K_{3}, K_{4} ; M_{1}, M_{2}, m_{1}, m_{2}\right] \\
& I_{4}\left(n_{3}, n_{2}, n_{1}, n_{4}\right)\left[K_{2}, K_{1}, K_{4}, K_{3} ; m_{1}, M_{2}, M_{1}, m_{2}\right] \\
& =\widehat{g}_{4}^{2} \widehat{g}_{2} I_{4}\left(n_{1}, n_{2}, n_{3}, n_{4}\right)\left[K_{1}, K_{2}, K_{3}, K_{4} ; M_{1}, M_{2}, m_{1}, m_{2}\right] \\
& I_{4}\left(n_{2}, n_{1}, n_{4}, n_{3}\right)\left[K_{1}, K_{4}, K_{3}, K_{2} ; M_{2}, M_{1}, m_{2}, m_{1}\right] \\
& =\widehat{g}_{4}^{3} \widehat{g}_{2} I_{4}\left(n_{1}, n_{2}, n_{3}, n_{4}\right)\left[K_{1}, K_{2}, K_{3}, K_{4} ; M_{1}, M_{2}, m_{1}, m_{2}\right]
\end{aligned}
$$

Furthermore, using the same idea in the subsection 3.1 we can write down similar recurrence relation of reduction coefficients for general $I_{4}\left(n_{1}, n_{2}, n_{3}, n_{4}\right)$ using the expansion of $I_{4}(2,1,1,1), I_{4}(1,2,1,1), I_{4}(1,1,2,1)$ and $I_{4}(1,1,1,2)$. However, by relation (4.12) and (4.13), all three cases $I_{4}(2,1,1,1), I_{4}(1,2,1,1)$ and $I_{4}(1,1,2,1)$ can be reduced to the reduction of $I_{4}(1,1,1,2)$, thus we need only to deal with the reduction of $I_{4}(1,1,1,2)$.

\subsection{Box $I_{4}(1,1,1,2)$}

Now, we use our method to calculate the reduction coefficients of $I_{4}(1,1,1,2)$. According our idea, we can write

$$
I_{4}(1,1,1,2)=\frac{\partial}{\partial m_{2}^{2}} I_{4}(1,1,1,1)
$$


Comparing with the calculation of triangle, we need to consider six different cuts: four cuts with nearby propagators and two with opposite propagators. By the symmetry, we could just calculate one for each type of cuts, and get the others by proper permutation. To demonstrate our method, let us show the calculation for the cut $K_{1}$ only. The reduction coefficients calculated for $I_{4}(1,1,1,2)$ has been checked using the LiteRed [23].

For the cut $K_{1}$, we have $K=K_{1}, P_{1}=K_{1}+K_{2}$ and $P_{2}=-K_{4}$ in (4.2). To calculate $\frac{\partial}{\partial m_{2}^{2}} C\left(I_{4}\right)$, since the parameter $\Delta$ and $b$ do not contain the $m_{2}$, we could just write it as

$$
\frac{\partial}{\partial m_{2}^{2}} C\left(I_{4}\right)=\left(\frac{\Delta}{4 K^{2}}\right)^{-\epsilon} \frac{b}{2 K^{2}} \frac{\partial}{\partial m_{2}^{2}} B o x^{(0)}
$$

To simplify $\frac{\partial}{\partial m_{2}^{2}} B o x^{(0)}$, first we rewrite $B o x^{(0)}$ as by partial integration and algebraic separation as

$$
\begin{aligned}
B o x^{(0)}=\frac{2}{-A} & {\left[(1+\epsilon) \int_{0}^{1} d u u^{-2-\epsilon} \sqrt{B-A u} \ln \left(\frac{D-C u+\sqrt{1-u} \sqrt{B-A u}}{D-C u-\sqrt{1-u} \sqrt{B-A u}}\right)\right.} \\
& +\frac{\epsilon C_{Z_{1}}}{Z_{1}\left(Z_{1}^{2}-1\right)} \operatorname{Tri}^{(0)}\left(Z_{1}\right)+\frac{\epsilon C_{Z_{2}}}{Z_{2}\left(Z_{2}^{2}-1\right)} \operatorname{Tri}^{(0)}\left(Z_{2}\right) \\
& \left.+\left(\frac{C_{Z_{1}}(1-2 \epsilon)}{Z_{1}^{2}-1}+\frac{C_{Z_{2}}(1-2 \epsilon)}{Z_{2}^{2}-1}\right) B u b^{(0)}\right]
\end{aligned}
$$

where the bubble and two triangles are specified by our cut $K$. Now we consider the action of $\frac{\partial}{\partial m_{2}^{2}}$. Since only $B$ and $D$ contains the parameter $m_{2}$, we can write directly

$$
\begin{aligned}
\frac{\partial}{\partial m_{2}^{2}} B o x^{(0)}= & \frac{2}{-A}\left[(1+\epsilon) \int_{0}^{1} d u u^{-2-\epsilon} \frac{B_{m_{2}^{2}}^{\prime}}{2 \sqrt{B-A u}} \ln \left(\frac{D-C u+\sqrt{1-u} \sqrt{B-A u}}{D-C u-\sqrt{1-u} \sqrt{B-A u}}\right)\right. \\
& +(1+\epsilon) \int_{0}^{1} d u u^{-2-\epsilon} \frac{(1-u)\left(u\left(2 A D_{\left(m_{2}^{2}\right)}^{\prime}-B_{\left(m_{2}^{2}\right)}^{\prime} C\right)+\left(B_{\left(m_{2}^{2}\right)}^{\prime} D-2 B D_{\left(m_{2}^{2}\right)}^{\prime}\right)\right)}{\sqrt{1-u}\left((D-C u)^{2}-(1-u)(B-A u)\right)} \\
& +\frac{\partial}{\partial m_{2}^{2}}\left(\frac{\epsilon C_{Z_{1}}}{Z_{1}\left(Z_{1}^{2}-1\right)}\right) \operatorname{Tri}^{(0)}\left(Z_{1}\right)+\frac{\partial}{\partial m_{2}^{2}}\left(\frac{\epsilon C_{Z_{2}}}{Z_{2}\left(Z_{2}^{2}-1\right)}\right) \operatorname{Tri}^{(0)}\left(Z_{2}\right) \\
& +\frac{\epsilon C_{Z_{1}}}{Z_{1}\left(Z_{1}^{2}-1\right)} \frac{\partial}{\partial m_{2}^{2}} \operatorname{Tri}^{(0)}\left(Z_{1}\right)+\frac{\epsilon C_{Z_{2}}}{Z_{2}\left(Z_{2}^{2}-1\right)} \frac{\partial}{\partial m_{2}^{2}} \operatorname{Tri}^{(0)}\left(Z_{2}\right) \\
& \left.+\frac{\partial}{\partial m_{2}^{2}}\left(\frac{C_{Z_{1}}(1-2 \epsilon)}{Z_{1}^{2}-1}+\frac{C_{Z_{2}}(1-2 \epsilon)}{Z_{2}^{2}-1}\right) B u b^{(0)}\right]
\end{aligned}
$$

where the $B_{\left(m_{2}^{2}\right)}^{\prime}$ and $D_{\left(m_{2}^{2}\right)}^{\prime}$ means $\frac{\partial}{\partial m_{2}^{2}} B$ and $\frac{\partial}{\partial m_{2}^{2}} D$. There are five terms in the bracket. Since the third line and fifth line are just some simple differentiation over coefficients, we calculate the first, second and four lines only:

- (1) For the first term, it is $\frac{(1+\epsilon) B_{\left(m_{2}^{2}\right)}^{\prime}}{2} B o x^{(-1)}$, while using (4.8), we can expand 


$$
\begin{aligned}
& B o x^{(-1)} \text { as }
\end{aligned}
$$



- (2) For the second term, using the trick of splitting terms and the recurrence relation, we get

$$
C_{3 ; Z_{1}} \operatorname{Tri}^{(0)}\left(Z_{1}\right)+C_{3 ; Z_{2}} \operatorname{Tri}^{(0)}\left(Z_{2}\right)+C_{2} B u b^{(0)}
$$

with

$$
\begin{aligned}
C_{3 ; Z_{1} ; K_{1}} & =\frac{\epsilon Z_{1}\left(\frac{\partial B}{\partial m_{2}^{2}} D-2 B \frac{\partial D}{\partial m_{2}^{2}}+\left(Z_{1}^{2}-1\right)\left(\frac{\partial B}{\partial m_{2}^{2}} C-2 A \frac{\partial D}{\partial m_{2}^{2}}\right)\right)}{\beta_{1} \beta_{2}\left(Z_{1}^{2}-1\right)^{2}\left(Z_{1}^{2}-Z_{2}^{2}\right)} \\
C_{3 ; Z_{2} ; K_{2}} & =\frac{\epsilon Z_{2}\left(\left(\frac{\partial B}{\partial m_{2}^{2}} D-2 B \frac{\partial D}{\partial m_{2}^{2}}\right)+\left(Z_{2}^{2}-1\right)\left(\frac{\partial B}{\partial m_{2}^{2}} C-2 A \frac{\partial D}{\partial m_{2}^{2}}\right)\right)}{\beta_{1} \beta_{2}\left(Z_{2}^{2}-1\right)^{2}\left(Z_{2}^{2}-Z_{1}^{2}\right)} \\
C_{2 ; K_{1}} & =\frac{n_{2 ; K_{1}}}{2 \beta_{1} \beta_{2}\left(Z_{1}^{2}-1\right)^{2}\left(Z_{2}^{2}-1\right)^{2}(\epsilon+1)}
\end{aligned}
$$

where

$$
\begin{aligned}
n_{2 ; K_{1}}= & (2 \epsilon-1)\left(2 \frac{\partial B}{\partial m_{2}^{2}} C\left(Z_{1}^{2}-1\right)\left(Z_{2}^{2}-1\right)(1+\epsilon)-4 A \frac{\partial D}{\partial m_{2}^{2}}\left(Z_{1}^{2}-1\right)\left(Z_{2}^{2}-1\right)(1+\epsilon)\right. \\
& +\frac{\partial B}{\partial m_{2}^{2}} D\left(-3-4 \epsilon+Z_{2}^{2}(1+2 \epsilon)+Z_{1}^{2}\left(1+Z_{2}^{2}+2 \epsilon\right)\right) \\
& \left.-2 B \frac{\partial D}{\partial m_{2}^{2}}\left(-3-4 \epsilon+Z_{2}^{2}(1+2 \epsilon)+Z_{1}^{2}\left(1+Z_{2}^{2}+2 \epsilon\right)\right)\right)
\end{aligned}
$$

- (3) For the fourth line with $\frac{\partial}{\partial m_{2}^{2}} \operatorname{Tr} i^{(0)}$, we could use result (3.32) and (3.28) to rewrite it as

$$
\begin{aligned}
&- \frac{\partial Z_{1}}{\partial m_{2}^{2}} \frac{2 \epsilon^{2} C_{Z_{1}}}{\left(Z_{1}^{2}-1\right)^{2}} \operatorname{Tri}^{(0)}\left(Z_{1}\right)-\frac{\partial Z_{2}}{\partial m_{2}^{2}} \frac{2 \epsilon^{2} C_{Z_{2}}}{\left(1-Z_{2}^{2}\right)^{2}} \operatorname{Tri}^{(0)}\left(Z_{2}\right) \\
&-\left(\frac{\partial Z_{1}}{\partial m_{2}^{2}} \frac{2 \epsilon(1-2 \epsilon) C_{Z_{1}}}{Z_{1}\left(Z_{1}^{2}-1\right)^{2}}+\frac{\partial Z_{2}}{\partial m_{2}^{2}} \frac{2 \epsilon(1-2 \epsilon) C_{Z_{2}}}{Z_{2}\left(Z_{2}^{2}-1\right)^{2}}\right) B u b^{(0)}
\end{aligned}
$$

Now collecting all results together for (4.17) we get our final result in the cut $K_{1}$ :

$$
\frac{\partial}{\partial m_{2}^{2}} I_{4}=c_{4 \rightarrow 4 ; K_{1}} I_{4}+c_{4 \rightarrow 3 ; \overline{4} ; K_{1}} I_{3 ; \overline{4}}+c_{4 \rightarrow 3, \overline{3} ; K_{1}} I_{3 ; \overline{3}} I+c_{4 \rightarrow 2 ; 12 ; K_{1}} I_{2 ; 12 ; K_{1}}+\cdots
$$

where

$$
I_{3 ; \overline{4}}=I_{4}(1,1,1,0), \quad I_{3 ; \overline{3}}=I_{4}(1,1,0,1), \quad I_{2 ; 12 ; K_{1}}=I_{4}(1,1,0,0)
$$

with the coefficients

$$
\begin{aligned}
& c_{4 \rightarrow 4 ; K_{1}}=-\frac{\frac{\partial B}{\partial m_{2}^{2}}\left(\frac{1}{2}+\epsilon\right)}{B} \\
& c_{4 \rightarrow 3 ; \overline{4} ; K_{1}}=\frac{-b \sqrt{\Delta_{3 ; m=0}\left[Z_{1}\right]}}{2 K_{1}^{2}}\left(\frac{-\epsilon C_{Z_{1}} \frac{\partial B}{\partial m_{2}^{2}}}{A B Z_{1}\left(1-Z_{1}^{2}\right)}-\frac{2(1+\epsilon)}{A} C_{3 ; Z_{1}}-\frac{2}{A} \frac{\partial}{\partial m_{2}^{2}}\left(\frac{\epsilon C_{Z_{1}}}{Z_{1}\left(Z_{1}^{2}-1\right)}\right)+\frac{2}{A} \frac{\partial Z_{1}}{\partial m_{2}^{2}} \frac{2 \epsilon^{2} C_{Z_{1}}}{\left(Z_{1}^{2}-1\right)^{2}}\right)
\end{aligned}
$$




$$
\begin{aligned}
c_{4 \rightarrow 3 ; \overline{3} ; K_{1}}= & \frac{-b \sqrt{\Delta_{3 ; m=0}\left[Z_{2}\right]}}{2 K_{1}^{2}}\left(\frac{-\epsilon C_{Z_{2}} \frac{\partial B}{\partial m_{2}^{2}}}{A B Z_{2}\left(1-Z_{2}^{2}\right)}-\frac{2(1+\epsilon)}{A} C_{3 ; Z_{2}}-\frac{2}{A} \frac{\partial}{\partial m_{2}^{2}}\left(\frac{\epsilon C_{Z_{2}}}{Z_{2}\left(Z_{2}^{2}-1\right)}\right)+\frac{2}{A} \frac{\partial Z_{2}}{\partial m_{2}^{2}} \frac{2 \epsilon^{2} C_{Z_{2}}}{\left(1-Z_{2}^{2}\right)^{2}}\right) \\
c_{4 \rightarrow 2 ; 12 ; K_{1}}= & \frac{1}{2 K_{1}^{2}}\left[-(1-2 \epsilon)\left(\frac{\frac{\partial B}{\partial m_{2}^{2}} C_{Z_{1}}}{A B\left(1-Z_{1}^{2}\right)}+\frac{\frac{\partial B}{\partial m_{2}^{2}} C_{Z_{2}}}{A B\left(1-Z_{2}^{2}\right)}\right)-\frac{2(1+\epsilon)}{A} C_{2}\right. \\
& \left.+\frac{2}{A}\left(\frac{\partial Z}{\partial m_{2}^{2}} \frac{2 \epsilon(1-2 \epsilon) C_{Z_{1}}}{Z_{1}\left(Z_{1}^{2}-1\right)^{2}}+\frac{\partial Z}{\partial m_{2}^{2}} \frac{2 \epsilon(1-2 \epsilon) C_{Z_{2}}}{Z_{2}\left(Z_{2}^{2}-1\right)^{2}}\right)-\frac{2}{A} \frac{\partial}{\partial m_{2}^{2}}\left(\frac{C_{Z_{1}}(1-2 \epsilon)}{Z_{1}^{2}-1}+\frac{C_{Z_{2}}(1-2 \epsilon)}{Z_{2}^{2}-1}\right)\right]
\end{aligned}
$$

where $C_{3 ; Z_{1}}, C_{3 ; Z_{2}}$ and $C_{2}$ given in eq. (4.20), and

$$
\Delta_{3 ; m=0}\left[Z_{1}\right]=4\left(\left(K_{1} \cdot K_{34}\right)^{2}-K_{1}^{2} K_{34}^{2}\right), \quad \Delta_{3 ; m=0}\left[Z_{2}\right]=4\left(\left(K_{1} \cdot K_{4}\right)^{2}-K_{1}^{2} K_{4}^{2}\right)
$$

Having given details for the cut $K_{1}$, the computation of other cuts will be similar, as shown in the section of triangle. For simplicity we will not list them one by one. All coefficients have been checked using LiteRed [23].

\section{Pentagon}

For the pentagon, let us define

$$
\begin{aligned}
& I_{5}\left(n_{1}, n_{2}, n_{3}, n_{4}, n_{5}\right)\left[K_{1}, K_{2}, K_{3}, K_{4}, K_{5} ; M_{1}, M_{2}, m_{1}, m_{2}, m_{3}\right] \\
& =\int \frac{d^{4-2 \epsilon} p}{(2 \pi)^{4-2 \epsilon}} \frac{1}{\left(p^{2}-M_{1}^{2}\right)^{n_{1}}\left(\left(p-K_{1}\right)^{2}-M_{2}^{2}\right)^{n_{2}}\left(\left(p-K_{12}\right)^{2}-m_{1}^{2}\right)^{n_{3}}\left(\left(p-K_{123}\right)^{2}-m_{2}^{2}\right)^{n_{4}}\left(\left(p+K_{5}\right)^{2}-m_{3}^{2}\right)^{n_{5}}} \\
& =\int \frac{d^{4-2 \epsilon} p}{(2 \pi)^{4-2 \epsilon}} \frac{1}{\left(p^{2}-M_{1}^{2}\right)^{n_{1}}\left((p-K)^{2}-M_{2}^{2}\right)^{n_{2}}\left(\left(p-P_{1}\right)^{2}-m_{1}^{2}\right)^{n_{3}}\left(\left(p-P_{2}\right)^{2}-m_{2}^{2}\right)^{n_{4}}\left(\left(p-P_{3}\right)^{2}-m_{3}^{2}\right)^{n_{5}}}
\end{aligned}
$$

where the second form is suitable for the discussion of unitarity cut with cut momentum $K$ in various cuts. The master basis of pentagon is given by $I_{5} \equiv I_{5}(1,1,1,1,1)$ and the cut part with $K$ is given by [9]

$$
\begin{aligned}
C\left(I_{5}\right)= & \left(\frac{\Delta}{4 K^{2}}\right)^{-\epsilon}(-b) \int_{0}^{1} d u u^{-1-\epsilon} \frac{\sqrt{1-u}}{\left(K^{2}\right)^{2}}\left(\frac{S\left[Q_{3}, Q_{2}, Q_{1}, K\right]}{4 \sqrt{\left(Q_{3} \cdot Q_{2}\right)^{2}-Q_{3}^{2} Q_{2}^{2}}} \ln \left(\frac{Q_{3} \cdot Q_{2}-\sqrt{\left(Q_{3} \cdot Q_{2}\right)^{2}-Q_{3}^{2} Q_{2}^{2}}}{Q_{3} \cdot Q_{2}+\sqrt{\left(Q_{3} \cdot Q_{2}\right)^{2}-Q_{3}^{2} Q_{2}^{2}}}\right)\right. \\
& +\frac{S\left[Q_{3}, Q_{1}, Q_{2}, K\right]}{4 \sqrt{\left(Q_{3} \cdot Q_{1}\right)^{2}-Q_{3}^{2} Q_{1}^{2}}} \ln \left(\frac{Q_{3} \cdot Q_{1}-\sqrt{\left(Q_{3} \cdot Q_{1}\right)^{2}-Q_{3}^{2} Q_{1}^{2}}}{Q_{3} \cdot Q_{1}+\sqrt{\left(Q_{3} \cdot Q_{1}\right)^{2}-Q_{3}^{2} Q_{1}^{2}}}\right) \\
& \left.+\frac{S\left[Q_{2}, Q_{1}, Q_{3}, K\right]}{4 \sqrt{\left(Q_{2} \cdot Q_{1}\right)^{2}-Q_{2}^{2} Q_{1}^{2}}} \ln \left(\frac{Q_{2} \cdot Q_{1}-\sqrt{\left(Q_{2} \cdot Q_{1}\right)^{2}-Q_{2}^{2} Q_{1}^{2}}}{Q_{2} \cdot Q_{1}+\sqrt{\left(Q_{2} \cdot Q_{1}\right)^{2}-Q_{2}^{2} Q_{1}^{2}}}\right)\right)
\end{aligned}
$$

where $Q_{i}=-(b \sqrt{1-u}) P_{i}+\frac{P_{i}^{2}+M_{1}^{2}-m_{i}^{2}-2 z\left(K \cdot P_{i}\right)}{K^{2}} K$ (see also $\left.(4.5)\right)$ and $S\left[Q_{3}, Q_{2}, Q_{1}, K\right]$ is a rational function defined as follows

$$
\begin{aligned}
S\left[Q_{j}, Q_{i}, Q_{k}, K\right] & =\frac{T_{j i k}}{T_{2}}, \quad T_{j i k}=-8 \operatorname{det}\left(\begin{array}{ccc}
Q_{k} \cdot K & Q_{j} \cdot K & Q_{i} \cdot K \\
Q_{j} \cdot Q_{k} & Q_{j}^{2} & Q_{j} \cdot Q_{i} \\
Q_{i} \cdot Q_{k} & Q_{j} \cdot Q_{i} & Q_{i}^{2}
\end{array}\right), \\
T_{2} & =-4 \operatorname{det}\left(\begin{array}{ccc}
Q_{3}^{2} & Q_{2} \cdot Q_{3} & Q_{1} \cdot Q_{3} \\
Q_{2} \cdot Q_{3} & Q_{2}^{2} & Q_{2} \cdot Q_{1} \\
Q_{1} \cdot Q_{3} & Q_{2} \cdot Q_{1} & Q_{1}^{2}
\end{array}\right)
\end{aligned}
$$


The form of $C\left(I_{5}\right)$ is like an addition of three different $C\left(I_{4}\right)$, with the common factor $\sqrt{1-u}$. To simplify further, noticing that there is a common factor $(1-u)^{2}$ between $T_{j i k}$ and $T_{2}$, we can define (please notice that $T_{j i k}=T_{i j k}$ )

$$
T_{2, r e}=\frac{T_{2}}{(1-u)^{2}}=H_{0}+H_{1} u, \quad T_{12}=\frac{T_{213}}{(1-u)^{2}}, \quad T_{13}=\frac{T_{312}}{(1-u)^{2}}, \quad T_{23}=\frac{T_{321}}{(1-u)^{2}}
$$

where $T_{i j}$ is independent of $u$ and $T_{2, r e}$ is a liner function of $u$. Furthermore, with parameters

$$
a=\frac{K^{2}+M_{1}^{2}-M_{2}^{2}}{K^{2}}, \quad b=\frac{\sqrt{\Delta\left[K, M_{1}, M_{2}\right]}}{K^{2}}, \quad a_{i} \equiv \frac{P_{i}^{2}+M_{1}^{2}-m_{i}^{2}}{K^{2}}
$$

we define

$\alpha_{i} \equiv a_{i} K^{2}-a P_{i} \cdot K, \quad \beta_{i} \equiv b^{2}\left(P_{i}^{2}-\frac{\left(P_{i} \cdot K\right)^{2}}{K^{2}}\right), \quad \gamma_{i j} \equiv b^{2}\left(\left(P_{i} \cdot P_{j}\right)^{2}-\frac{\left(P_{i} \cdot K\right)\left(P_{j} \cdot K\right)}{K^{2}}\right)$

thus $A, B, C, D$ defined in (4.4) for box cut and (5.3) can be simplified as

$$
\begin{array}{ll}
A_{12}=\gamma_{12}^{2}-\beta_{1} \beta_{2}, & B_{12}=\frac{-\alpha_{2}^{2} \beta_{1}-\alpha_{1}^{2} \beta_{2}+2 \alpha_{1} \alpha_{2} \gamma_{12}}{K^{2}}+\gamma_{12}^{2}-\beta_{1} \beta_{2}, \\
C_{12}=\gamma_{12}, & D_{12}=\gamma_{12}+\frac{\alpha_{1} \alpha_{2}}{K^{2}}
\end{array}
$$

and

$$
\begin{aligned}
H_{1}= & 4 \beta_{1} \beta_{2} \beta_{3}+8 \gamma_{12} \gamma_{13} \gamma_{23}-4 \beta_{3} \gamma_{12}^{2}-4 \beta_{2} \gamma_{13}^{2}-4 \beta_{1} \gamma_{23}^{2} \\
H_{0}= & -H_{1}+\frac{4}{K^{2}}\left(\alpha_{1}^{2} A_{23}+\alpha_{2}^{2} A_{13}+\alpha_{3}^{2} A_{12}\right) \\
& +8\left(\left(D_{23}-C_{23}\right)\left(\beta_{1} C_{23}-C_{12} C_{13}\right)+\left(D_{13}-C_{13}\right)\left(\beta_{2} C_{13}-C_{12} C_{23}\right)\right. \\
& \left.+\left(D_{12}-C_{12}\right)\left(\beta_{3} C_{12}-C_{13} C_{23}\right)\right) \\
T_{12}= & 8\left(\alpha_{3} A_{12}+\alpha_{1} \beta_{2} \gamma_{13}-\alpha_{2} \gamma_{12} \gamma_{13}+\alpha_{2} \beta_{1} \gamma_{23}-\alpha_{1} \gamma_{12} \gamma_{23}\right) \\
T_{23}= & 8\left(\alpha_{1} A_{23}+\alpha_{2} \beta_{3} \gamma_{12}+\alpha_{3} \beta_{2} \gamma_{13}-\alpha_{3} \gamma_{12} \gamma_{23}-\alpha_{2} \gamma_{13} \gamma_{23}\right) \\
T_{13}= & 8\left(\alpha_{2} A_{13}+\alpha_{1} \beta_{3} \gamma_{12}+\alpha_{3} \beta_{1} \gamma_{23}-\alpha_{1} \gamma_{13} \gamma_{23}-\alpha_{3} \gamma_{12} \gamma_{13}\right)
\end{aligned}
$$

With above new notations, we can rewrite the expression of $C\left(I_{5}\right)$ as

$$
\begin{aligned}
C\left(I_{5}\right)= & \left(\frac{\Delta}{4 K^{2}}\right)^{-\epsilon} \frac{b}{4\left(K^{2}\right)^{2}} \int_{0}^{1} d u u^{-1-\epsilon} \\
& \times \frac{1}{H_{0}+H_{1} u}\left\{\frac{T_{23}}{\sqrt{B_{23}-A_{23} u}} \ln \left(\frac{D_{23}-C_{23} u+\sqrt{1-u} \sqrt{B_{23}-A_{23} u}}{D_{23}-C_{23} u-\sqrt{1-u} \sqrt{B_{23}-A_{23} u}}\right)\right. \\
& +\frac{T_{13}}{\sqrt{B_{13}-A_{13} u}} \ln \left(\frac{D_{13}-C_{13} u+\sqrt{1-u} \sqrt{B_{13}-A_{13} u}}{D_{13}-C_{13} u-\sqrt{1-u} \sqrt{B_{13}-A_{13} u}}\right) \\
& \left.+\frac{T_{12}}{\sqrt{B_{12}-A_{12} u}} \ln \left(\frac{D_{12}-C_{12} u+\sqrt{1-u} \sqrt{B_{12}-A_{12} u}}{D_{12}-C_{12} u-\sqrt{1-u} \sqrt{B_{12}-A_{12} u}}\right)\right\}
\end{aligned}
$$


We can see that in (5.8) only parameters $\alpha_{i}$ contains $m_{i}^{2}$, so

$$
\frac{\partial}{\partial m_{i}^{2}}=\frac{\partial}{\partial \alpha_{i}} \frac{\partial \alpha_{i}}{\partial a_{i}} \frac{\partial a_{i}}{\partial m_{i}^{2}}=-\frac{\partial}{\partial \alpha_{i}}
$$

For later convenience, we define three functions

$$
\begin{aligned}
\operatorname{pen}_{i j}^{(n)} & =\int_{0}^{1} d u u^{n-1-\epsilon} \frac{1}{H_{0}+H_{1} u} \frac{1}{\sqrt{B_{i j}-A_{i j} u}} \ln \left(\frac{D_{i j}-C_{i j} u+\sqrt{1-u} \sqrt{B_{i j}-A_{i j} u}}{D_{i j}-C_{i j} u-\sqrt{1-u} \sqrt{B_{i j}-A_{i j} u}}\right) \\
& =\int_{0}^{1} d u u^{n-1-\epsilon} \frac{1}{H_{0}+H_{1} u} \frac{1}{\sqrt{B_{i j}-A_{i j} u}} \ln \left(*_{i j}\right), \quad *_{i j} \\
& \equiv \frac{D_{i j}-C_{i j} u+\sqrt{1-u} \sqrt{B_{i j}-A_{i j} u}}{D_{i j}-C_{i j} u-\sqrt{1-u} \sqrt{B_{i j}-A_{i j} u}}
\end{aligned}
$$

thus the $C\left(I_{5}\right)$ could be written as

$$
C\left(I_{5}\right)=\left(\frac{\Delta}{4 K^{2}}\right)^{-\epsilon} \frac{b}{4\left(K^{2}\right)^{2}}\left(T_{23} \text { pen }_{23}^{(0)}+T_{13} \text { pen }_{13}^{(0)}+T_{12} \text { pen }_{12}^{(0)}\right)
$$

From the definition of (5.10), one can easily establish the recursion relation by rewriting $u=\frac{\left(H_{0}+H_{1} u\right)-H_{0}}{H_{1}}$. The cancelation of denominator $H_{0}+H_{1} u$ in (5.10) is nothing, but the cut of corresponding box (see (4.6)).

Similar to other sections, with momentum shifting and reflection, we can define the action

$$
\begin{aligned}
\widehat{g}_{5}: & \left\{\left(n_{1}, n_{2}, n_{3}, n_{4}, n_{5}\right) ;\left(K_{1}, K_{2}, K_{3}, K_{4}, K_{5}\right) ;\left(M_{1}, M_{2}, m_{1}, m_{2}, m_{3}\right)\right\} \\
\rightarrow & \left\{\left(n_{2}, n_{3}, n_{4}, n_{5}, n_{1}\right) ;\left(K_{2}, K_{3}, K_{4}, K_{5}, K_{1}\right) ;\left(M_{2}, m_{1}, m_{2}, m_{3}, M_{1}\right)\right\} \\
\widehat{g}_{2}: \quad & \left\{\left(n_{1}, n_{2}, n_{3}, n_{4}, n_{5}\right) ;\left(K_{1}, K_{2}, K_{3}, K_{4}, K_{5}\right) ;\left(M_{1}, M_{2}, m_{1}, m_{2}, m_{3}\right)\right\} \\
\rightarrow & \left\{\left(n_{2}, n_{1}, n_{5}, n_{4}, n_{3}\right) ;\left(K_{1}, K_{5}, K_{4}, K_{3}, K_{2}\right) ;\left(M_{2}, M_{1}, m_{3}, m_{2}, m_{1}\right)\right\}
\end{aligned}
$$

thus if we write $\widehat{I}_{5} \equiv I_{5}\left(n_{1}, n_{2}, n_{3}, n_{4}, n_{5}\right)\left[K_{1}, K_{2}, K_{3} K_{4}, K_{5} ; M_{1}, M_{2}, m_{1}, m_{2}, m_{3}\right]$ we will have

$$
\begin{aligned}
& I_{5}\left(n_{2}, n_{3}, n_{4}, n_{5}, n_{1}\right)\left[K_{2}, K_{3}, K_{4}, K_{5}, K_{1} ; M_{2}, m_{1}, m_{2}, m_{3}, M_{1}\right]=\widehat{g}_{5} \widehat{I}_{5} \\
& I_{5}\left(n_{3}, n_{4}, n_{5}, n_{1}, n_{2}\right)\left[K_{3}, K_{4}, K_{5}, K_{1}, K_{2} ; m_{1}, m_{2}, m_{3}, M_{1}, M_{2}\right]=\widehat{g}_{5}^{2} \widehat{I}_{5} \\
& I_{5}\left(n_{4}, n_{5}, n_{1}, n_{2}, n_{3}\right)\left[K_{4}, K_{5}, K_{1}, K_{2}, K_{3} ; m_{2}, m_{3}, M_{1}, M_{2}, m_{1}\right]=\widehat{g}_{5}^{3} \widehat{I}_{5} \\
& I_{5}\left(n_{5}, n_{1}, n_{2}, n_{3}, n_{4}\right)\left[K_{5}, K_{1}, K_{2}, K_{3}, K_{4} ; m_{3}, M_{1}, M_{2}, m_{1}, m_{2}\right]=\widehat{g}_{5}^{4} \widehat{I}_{5}
\end{aligned}
$$

and

$$
\begin{aligned}
& I_{5}\left(n_{2}, n_{1}, n_{5}, n_{4}, n_{3}\right)\left[K_{1}, K_{5}, K_{4}, K_{3}, K_{2} ; M_{2}, M_{1}, m_{3}, m_{2}, m_{1}\right]=\widehat{g}_{2} \widehat{I}_{5} \\
& I_{5}\left(n_{3}, n_{2}, n_{1}, n_{5}, n_{4}\right)\left[K_{2}, K_{1}, K_{5}, K_{4}, K_{3} ; m_{1}, M_{2}, M_{1}, m_{3}, m_{2}\right]=\widehat{g}_{5}^{-1} \widehat{g}_{2} \widehat{I}_{5} \\
& I_{5}\left(n_{4}, n_{3}, n_{2}, n_{1}, n_{5}\right)\left[K_{3}, K_{2}, K_{1}, K_{5}, K_{4} ; m_{2}, m_{1}, M_{2}, M_{1}, m_{3}\right]=\widehat{g}_{5}^{-2} \widehat{g}_{2} \widehat{I}_{5} \\
& I_{5}\left(n_{5}, n_{4}, n_{3}, n_{2}, n_{1}\right)\left[K_{4}, K_{3}, K_{2}, K_{1}, K_{5} ; m_{3}, m_{2}, m_{1}, M_{2}, M_{1}\right]=\widehat{g}_{5}^{-3} \widehat{g}_{2} \widehat{I}_{5} \\
& I_{5}\left(n_{1}, n_{5}, n_{4}, n_{3}, n_{2}\right)\left[K_{5}, K_{4}, K_{3}, K_{2}, K_{1} ; M_{1}, m_{3}, m_{2}, m_{1}, M_{2}\right]=\widehat{g}_{5}^{-4} \widehat{g}_{2} \widehat{I}_{5}
\end{aligned}
$$


Furthermore, using the same idea in the subsection 3.1 we can write down similar recurrence relation for general $I_{5}\left(n_{1}, n_{2}, n_{3}, n_{4}, n_{5}\right)$ using the expansion of $I_{5}(2,1,1,1,1)$, $I_{5}(1,2,1,1,1), I_{5}(1,1,2,1,1), I_{5}(1,1,1,2,1)$ and $I_{5}(1,1,1,1,2)$. However, by relation (5.13) and (5.14), all other four cases can be reduced to the reduction of $I_{5}(1,1,1,1,2)$.

To reduce $I_{5}(1,1,1,1,2)$, according to our ideas, we should calculate $\frac{\partial}{\partial m_{3}^{2}} I_{5}(1,1,1,1,1)$ in $\left(\begin{array}{l}5 \\ 2\end{array}\right)=10$ different cuts. Again, in the main part, we present only the computation of the cut $K_{1}$. For this case, since the analytic checking using LiteRed [23] is too hard, we have checked only numerically.

\subsection{Cut $K_{1}$ of $I_{2}(1,1,1,1,2)$}

For this cut, we will choose the parameters of the second form in (5.1) as $K=K_{1}, P_{1}=K_{12}$, $P_{2}=K_{123}$ and $P_{3}=-K_{5}$. Since the $m_{3}$ is not contained in $\left(\frac{\Delta}{4 K^{2}}\right)^{-\epsilon}$, we could just drop the factor $\left(\frac{\Delta}{4 K^{2}}\right)^{-\epsilon}$. Furthermore the parameter $m_{3}$ is only contained in $B, D, H_{0}$ and $T_{12}$, $T_{13}$ and $T_{23}$, thus we have

$$
\begin{aligned}
\frac{\partial}{\partial m_{3}^{2}} C\left(I_{5}\right)=\frac{b}{4\left(K^{2}\right)^{2}} & \left(\frac{\partial T_{23}}{\partial m_{3}^{2}} \text { pen }_{23}^{(0)}+\frac{\partial T_{13}}{\partial m_{3}^{2}} \text { pen }_{13}^{(0)}+\frac{\partial T_{12}}{\partial m_{3}^{2}} \text { pen }_{12}^{(0)}\right. \\
& \left.+T_{23} \frac{\partial}{\partial m_{3}^{2}} \text { pen }_{23}^{(0)}+T_{13} \frac{\partial}{\partial m_{3}^{2}} \text { pen }_{13}^{(0)}+T_{12} \frac{\partial}{\partial m_{3}^{2}} \text { pen }_{12}^{(0)}\right)
\end{aligned}
$$

The result of $\frac{\partial T_{i j}}{\partial m_{3}^{2}}$ is very simple and given by

$$
\frac{\partial T_{23}}{\partial m_{3}^{2}}=8\left(\gamma_{12} \gamma_{23}-\beta_{2} \gamma_{13}\right), \quad \frac{\partial T_{13}}{\partial m_{3}^{2}}=8\left(\gamma_{12} \gamma_{13}-\beta_{1} \gamma_{23}\right), \quad \frac{\partial T_{12}}{\partial m_{3}^{2}}=8\left(\beta_{1} \beta_{2}-\gamma_{12}^{2}\right)
$$

To calculate $\frac{\partial}{\partial m_{3}^{2}}$ pen $_{i j}^{(0)}$, noticing that

$$
\begin{aligned}
\frac{\partial}{\partial m_{3}^{2}} \text { pen }^{(0)}= & \int_{0}^{1} d u u^{-1-\epsilon} \frac{1}{\left(H_{0}+H_{1} u\right)^{2}} \frac{-\frac{\partial H_{0}}{\partial m_{3}^{2}}}{\sqrt{B-A u}} \ln (*) \\
& +\int_{0}^{1} d u u^{-1-\epsilon} \frac{1}{H_{0}+H_{1} u} \frac{-\frac{1}{2} \frac{\partial B}{\partial m_{3}^{2}}}{\sqrt{B-A u}} \ln (*) \\
& +\int_{0}^{1} d u u^{-1-\epsilon} \frac{1}{H_{0}+H_{1} u} \frac{1}{\sqrt{B-A u}} \frac{\partial}{\partial m_{3}^{2}} \ln (*) \equiv L_{1}+L_{2}+L_{3}
\end{aligned}
$$

we need to calculate these three terms respectively.

To prepare the reduction of three integral $L_{1}, L_{2}$, and $L_{3}$, we rewrite pen ${ }^{(n)}$, which is defined in (5.10), as following To prepare the reduction of three integral $L_{1}, L_{2}$, and $L_{3}$, we want to rewrite pen ${ }^{(n)}$ defined in (5.10) in the following by doing the partial integration $d u^{n-\epsilon}=(n-\epsilon) u^{n-1-\epsilon} d u$

$$
\begin{aligned}
\text { pen }^{(n)}=\frac{1}{n-\epsilon} & \left\{H_{1} \int_{0}^{1} d u u^{n-\epsilon} \frac{1}{\left(H_{0}+H_{1} u\right)^{2}} \frac{1}{\sqrt{B-A u}} \ln (*)\right. \\
- & \frac{A}{2} \int_{0}^{1} d u u^{n-\epsilon} \frac{1}{H_{0}+H_{1} u} \frac{1}{\sqrt{B-A u}^{3}} \ln (*) \\
& \left.+\int_{0}^{1} d u u^{n-\epsilon} \frac{A D+B D-2 B C+u(A C+B C-2 A D)}{\left(H_{0}+H_{1} u\right)(B-A u)} \frac{1}{\sqrt{1-u} \$}\right\}
\end{aligned}
$$


where

$$
\$_{i j}=\left(D_{i j}-C_{i j} u\right)^{2}-(1-u)\left(B_{i j}-A_{i j} u\right)=\beta_{i} \beta_{j}\left(1-u-Z_{i}^{2}\right)\left(1-u-Z_{j}^{2}\right)
$$

The first line in (5.18) has the form of $L_{1}$ in (5.17) since $H_{0}$ does not depend on $u$ as defined in (5.3).

Now we consider the third line in (5.18). Using algebraic separation it becomes

$$
\begin{aligned}
\int_{0}^{1} d u u^{n-\epsilon} \frac{A D+B D-2 B C+u(A C+B C-2 A D)}{\left(H_{0}+H_{1} u\right)(B-A u)} \frac{1}{\sqrt{1-u} \$} \\
=\frac{(A D+B D-2 B C) H_{1}-H_{0}(A C+B C-2 A D)}{A H_{0}+B H_{1}} \int_{0}^{1} d u u^{n-\epsilon} \frac{1}{\left(H_{0}+H_{1} u\right) \sqrt{1-u} \$} \\
\quad+\frac{(A D+B D-2 B C) A+B(A C+B C-2 A D)}{A H_{0}+B H_{1}} \int_{0}^{1} d u u^{n-\epsilon} \frac{1}{(B-A u) \sqrt{1-u} \$}
\end{aligned}
$$

Among these two terms in (5.20), the second term will be canceled by the second line in (5.18). For the first term, using the factorization form of $\$_{i j}$ in (5.19) we get

$$
\begin{gathered}
\frac{1}{\left(H_{0}+H_{1} u\right) \sqrt{1-u} \Phi_{i j}}=\frac{1}{\sqrt{1-u} \beta_{i} \beta_{j}\left(Z_{i}^{2}-Z_{j}^{2}\right)} \times \frac{1}{H_{0}+H_{1} u}\left(\frac{1}{1-u-Z_{i}^{2}}-\frac{1}{1-u-Z_{j}^{2}}\right) \\
=W_{0 ; i j} \frac{1}{\sqrt{1-u}\left(H_{0}+H_{1} u\right)}+W_{i ; i j} \frac{1}{\sqrt{1-u}\left(1-u-Z_{i}^{2}\right)}+W_{j ; i j} \frac{1}{\sqrt{1-u}\left(1-u-Z_{j}^{2}\right)}
\end{gathered}
$$

with the coefficients

$$
\begin{aligned}
& W_{0 ; i j}=\frac{1}{\beta_{i} \beta_{j}\left(Z_{i}^{2}-Z_{j}^{2}\right)}\left(\frac{H_{1}}{H_{0}+\left(1-Z_{i}^{2}\right) H_{1}}-\frac{H_{1}}{H_{0}+\left(1-Z_{j}^{2}\right) H_{1}}\right) \\
& W_{i ; i j}=\frac{1}{\beta_{i} \beta_{j}\left(Z_{i}^{2}-Z_{j}^{2}\right)} \frac{1}{H_{0}+\left(1-Z_{i}^{2}\right) H_{1}}, \quad W_{j ; i j}=\frac{1}{\beta_{i} \beta_{j}\left(Z_{i}^{2}-Z_{j}^{2}\right)} \frac{-1}{H_{0}+\left(1-Z_{j}^{2}\right) H_{1}}
\end{aligned}
$$

Among three terms in (5.21), the first term is spurious and will be canceled when summing contributions from three pen $_{i j}$ terms. The second and third terms are essentially the triangle part. Putting all coefficients back, we find the third line in (5.18) is given by

$$
\begin{aligned}
\int_{0}^{1} d u u^{n-\epsilon} \frac{A D+B D-2 B C+u(A C+B C-2 A D)}{\left(H_{0}+H_{1} u\right)(B-A u)} \frac{1}{\sqrt{1-u} \$} \\
=\frac{(A-B)(A D-B C)}{A H_{0}+B H_{1}} \int_{0}^{1} d u u^{n-\epsilon} \frac{1}{(B-A u) \sqrt{1-u} \$} \\
\quad+G_{i j} W_{0 ; i j} \int_{0}^{1} d u u^{n-\epsilon} \frac{1}{\sqrt{1-u}\left(H_{0}+H_{1} u\right)}-\frac{G_{i j} W_{i ; i j}(n-\epsilon)}{Z_{i}} \operatorname{Tri}^{(n)}\left(Z_{i}\right) \\
\quad-\frac{G_{i j} W_{j ; i j}(n-\epsilon)}{Z_{j}} \operatorname{Tri}^{(n)}\left(Z_{j}\right)
\end{aligned}
$$

where

$$
G_{i j} \equiv \frac{\left(A_{i j} D_{i j}+B_{i j} D_{i j}-2 B_{i j} C_{i j}\right) H_{1}-H_{0}\left(A_{i j} C_{i j}+B_{i j} C_{i j}-2 A_{i j} D_{i j}\right)}{\left(A_{i j} H_{0}+B_{i j} H_{1}\right)}
$$


For the second line in (5.18), we will use the rewriting of box

$$
\begin{aligned}
B o x^{(n)}=\frac{1}{n-\epsilon} \times & \left\{-\frac{A}{2} \int_{0}^{1} d u u^{n-\epsilon} \frac{1}{\sqrt{B-A u}^{3}} \ln (*)\right. \\
& \left.+\int_{0}^{1} d u u^{n-\epsilon} \frac{A D+B D-2 B C+u(A C+B C-2 A D)}{(B-A u) \sqrt{1-u} \$}\right\}
\end{aligned}
$$

which is obtained by the similar method as in (5.18). Using the similar splitting technique done in previous paragraphes to the second term in (5.25), we will arrive

$$
\begin{aligned}
B o x_{i j}^{(n)}= & \frac{1}{n-\epsilon}\left\{\frac{-A}{2} \int_{0}^{1} d u u^{n-\epsilon} \frac{1}{\sqrt{B-A u}^{3}} \ln (*)\right. \\
& +\frac{(A-B)(A D-B C)}{A} \int_{0}^{1} d u u^{n-\epsilon} \frac{1}{(B-A u) \sqrt{1-u} \$} \\
& -\frac{2 A D-A C-B C}{A} \frac{1}{\beta_{i} \beta_{j}\left(Z_{i}^{2}-Z_{j}^{2}\right)} \frac{n-\epsilon}{Z_{i}} \operatorname{Tri}^{(n)}\left(Z_{i}\right) \\
& \left.+\frac{2 A D-A C-B C}{A} \frac{1}{\beta_{i} \beta_{j}\left(Z_{i}^{2}-Z_{j}^{2}\right)} \frac{n-\epsilon}{Z_{j}} \operatorname{Tri}^{(n)}\left(Z_{j}\right)\right\}
\end{aligned}
$$

For the first term in (5.26), we do following manipulation

$$
\begin{aligned}
\int_{0}^{1} d u u^{n-\epsilon} \frac{1}{\sqrt{B-A u}^{3}} \ln (*) & =\int_{0}^{1} d u u^{n-\epsilon} \frac{H_{0}+H_{1} u}{\left(H_{0}+H_{1} u\right) \sqrt{B-A u}^{3}} \ln (*) \\
& =\int_{0}^{1} d u u^{n-\epsilon} \frac{H_{0}+H_{1} \frac{A u-B+B}{A}}{\left(H_{0}+H_{1} u\right) \sqrt{B-A u}^{3}} \ln (*) \\
& =\left(H_{0}+\frac{B H_{1}}{A}\right) \int_{0}^{1} d u u^{n-\epsilon} \frac{1}{\left(H_{0}+H_{1} u\right) \sqrt{B-A u}^{3}} \ln (*)-\frac{H_{1}}{A} \text { pen }^{(n+1)}
\end{aligned}
$$

Thus the second line in (5.18) becomes

$$
\begin{aligned}
-\frac{A}{2} \int_{0}^{1} d u u^{n-\epsilon} \frac{1}{\left(H_{0}+H_{1} u\right) \sqrt{B-A u}^{3}} \ln (*)= & \frac{A(n-\epsilon)}{A H_{0}+B H_{1}} \operatorname{Box}^{(n)}-\frac{A H_{1}}{2\left(A H_{0}+B H_{1}\right)} \operatorname{pen}^{(n+1)} \\
& -\frac{(A-B)(A D-B C)}{\left(A H_{0}+B H_{1}\right)} \int_{0}^{1} d u u^{n-\epsilon} \frac{1}{(B-A u) \sqrt{1-u} \$} \\
& -\frac{A \lambda_{i ; i j}(n-\epsilon)}{2} \operatorname{Tri}^{(n)}\left(Z_{i}\right)-\frac{A \lambda_{j ; i j}(n-\epsilon)}{2} \operatorname{Tri}^{(n)}\left(Z_{j}\right)
\end{aligned}
$$

with the coefficients

$$
\begin{aligned}
\lambda_{i ; i j} & =\frac{2(A C+B C-2 A D)}{A\left(A H_{0}+B H_{1}\right)} \frac{1}{\beta_{i} \beta_{j}\left(Z_{i}^{2}-Z_{j}^{2}\right)} \frac{1}{Z_{i}}, \\
\lambda_{j ; i j} & =\frac{2(A C+B C-2 A D)}{A\left(A H_{0}+B H_{1}\right)} \frac{1}{\beta_{i} \beta_{j}\left(Z_{j}^{2}-Z_{i}^{2}\right)} \frac{1}{Z_{j}}
\end{aligned}
$$

Now we can explicitly see that the third term in (5.28) cancel the first term in (5.23). 
Putting all together, we have

$$
\begin{aligned}
\operatorname{pen}^{(n)}= & \frac{H_{1}}{n-\epsilon} \int_{0}^{1} d u u^{n-\epsilon} \frac{1}{\left(H_{0}+H_{1} u\right)^{2}} \frac{1}{\sqrt{B-A u}} \ln (*)+\frac{A}{A H_{0}+B H_{1}} B_{o x}^{(n)} \\
& -\frac{A H_{1}}{2\left(A H_{0}+B H_{1}\right)} \frac{1}{n-\epsilon} \operatorname{pen}^{(n+1)}-\left(\frac{A \lambda_{i ; i j}}{2}+\frac{G_{i j} W_{i ; i j}}{Z_{i}}\right) \operatorname{Tri}^{(n)}\left(Z_{i}\right) \\
& -\left(\frac{A \lambda_{j ; i j}}{2}+\frac{G_{i j} W_{j ; i j}}{Z_{j}}\right) \operatorname{Tri}^{(n)}\left(Z_{j}\right)+\frac{G_{i j} W_{0 ; i j}}{n-\epsilon} \int_{0}^{1} d u u^{n-\epsilon} \frac{1}{\sqrt{1-u}\left(H_{0}+H_{1} u\right)}
\end{aligned}
$$

with the coefficients given by (5.22), (5.24) and (5.29).

Now we can consider the $L_{1}, L_{2}, L_{3}$ terms. Let us start from the $L_{1}$ term. By comparing $L_{1}$ with the first term in (5.17), we see that

$$
\begin{aligned}
L_{1}= & -\frac{\partial H_{0}}{\partial m_{3}^{2}} \int_{0}^{1} d u u^{-1-\epsilon} \frac{1}{\left(H_{0}+H_{1} u\right)^{2} \sqrt{B-A u}} \ln (*) \\
= & \frac{\partial H_{0}}{\partial m_{3}^{2}} \frac{(1+\epsilon)}{H_{1}} \operatorname{pen}^{(-1)}-\frac{\partial H_{0}}{\partial m_{3}^{2}} \frac{A(1+\epsilon)}{H_{1}\left(A H_{0}+B H_{1}\right)} \operatorname{Box}^{(-1)}-\frac{\partial H_{0}}{\partial m_{3}^{2}} \frac{A}{2\left(A H_{0}+B H_{1}\right)} \text { pen }^{(0)} \\
& +\frac{\partial H_{0}}{\partial m_{3}^{2}}\left(\frac{A \lambda_{i ; i j}}{2}+\frac{G_{i j} W_{i ; i j}}{Z_{i}}\right) \frac{(1+\epsilon)}{H_{1}} \operatorname{Tri}^{(-1)}\left(Z_{i}\right) \\
& +\frac{\partial H_{0}}{\partial m_{3}^{2}}\left(\frac{A \lambda_{j ; i j}}{2}+\frac{G_{i j} W_{j ; i j}}{Z_{j}}\right) \frac{(1+\epsilon)}{H_{1}} \operatorname{Tri}^{(-1)}\left(Z_{j}\right) \\
& +\frac{\partial H_{0}}{\partial m_{3}^{2}} \frac{G_{i j} W_{0 ; i j}}{H_{1}} \int_{0}^{1} d u u^{-1-\epsilon} \frac{1}{\sqrt{1-u}\left(H_{0}+H_{1} u\right)}
\end{aligned}
$$

For the $L_{2}$, which is written as

$$
L_{2}=-\frac{1}{2} \frac{\partial B}{\partial m_{3}^{2}} \int_{0}^{1} d u u^{-1-\epsilon} \frac{1}{\left(H_{0}+H_{1} u\right) \sqrt{B-A u}^{3}} \ln (*)
$$

using the (5.27) with $n=-1$ we have

$$
\begin{aligned}
L_{2}= & -\frac{\partial B}{\partial m_{3}^{2}} \frac{(1+\epsilon)}{A H_{0}+B H_{1}} B^{(-1)}-\frac{1}{2} \frac{\partial B}{\partial m_{3}^{2}} \frac{H_{1}}{A H_{0}+B H_{1}} \text { pen }^{(0)} \\
& -\frac{\partial B}{\partial m_{3}^{2}} \frac{(A-B)(A D-B C)}{A\left(A H_{0}+B H_{1}\right)} \int_{0}^{1} d u u^{-1-\epsilon} \frac{1}{(B-A u) \sqrt{1-u} \$} \\
& +\frac{1}{2} \frac{\partial B}{\partial m_{3}^{2}} \lambda_{i ; i j}(1+\epsilon) \operatorname{Tri}^{(-1)}\left(Z_{i}\right)+\frac{1}{2} \frac{\partial B}{\partial m_{3}^{2}} \lambda_{j ; i j}(1+\epsilon) \operatorname{Tri}^{(-1)}\left(Z_{j}\right)
\end{aligned}
$$

For the integral $L_{3}$

$$
L_{3}=\int_{0}^{1} d u u^{-1-\epsilon} \frac{1}{\left(H_{0}+H_{1} u\right)} \frac{(1-u)\left[B^{\prime} D-2 B D^{\prime}+u\left(2 A D^{\prime}-B^{\prime} C\right)\right]}{(B-A u) \sqrt{1-u} \$}
$$

where $B^{\prime} \equiv \frac{\partial B}{\partial m_{3}^{2}}, D^{\prime} \equiv \frac{\partial D}{\partial m_{3}^{2}}$, using the splitting of $\S$ in (5.19) it is given by

$$
\begin{aligned}
L_{3}= & r_{1} \int_{0}^{1} d u u^{-1-\epsilon} \frac{1}{\sqrt{1-u} \$}+r_{2} \int_{0}^{1} d u u^{-1-\epsilon} \frac{1}{(B-A u) \sqrt{1-u} \$} \\
& +r_{3} \int_{0}^{1} d u u^{-1-\epsilon} \frac{1}{\left(H_{0}+H_{1} u\right) \sqrt{1-u} \$}
\end{aligned}
$$


with the coefficients

$$
\begin{aligned}
& r_{1}=\frac{2 A D^{\prime}-B^{\prime} C}{A H_{1}}, \quad r_{2}=\frac{B^{\prime}(A-B)(A D-B C)}{A\left(A H_{0}+B H_{1}\right)} \\
& r_{3}=\frac{\left(H_{0}+H_{1}\right)\left[\left(B^{\prime} D-2 B D^{\prime}\right) H_{1}-\left(2 A D^{\prime}-B^{\prime} C\right) H_{0}\right]}{H_{1}\left(A H_{0}+B H_{1}\right)}
\end{aligned}
$$

There are three terms. The first term could be split into two triangles, as we have done before. The second term is a spurious term, and is canceled with the same term in the integral $L_{2}$. And the last term could also be split into three pieces by using (5.23) with $n=-1$. Putting all together we have

$$
\begin{aligned}
L_{3}= & r_{2} \int_{0}^{1} d u u^{-1-\epsilon} \frac{1}{(B-A u) \sqrt{1-u} \$}+r_{3} W_{0 ; i j} \int_{0}^{1} d u u^{-1-\epsilon} \frac{1}{\sqrt{1-u}\left(H_{0}+H_{1} u\right)} \\
& +\left(\frac{r_{1}}{\beta_{i} \beta_{j}\left(Z_{i}^{2}-Z_{j}^{2}\right)}+r_{3} W_{i ; i j}\right) \frac{1+\epsilon}{Z_{i}} \operatorname{Tri}^{(-1)}\left(Z_{i}\right) \\
& +\left(\frac{r_{1}}{\beta_{i} \beta_{j}\left(Z_{j}^{2}-Z_{i}^{2}\right)}+r_{3} W_{j ; i j}\right) \frac{1+\epsilon}{Z_{j}} \operatorname{Tri}^{(-1)}\left(Z_{j}\right)
\end{aligned}
$$

Collecting above results for $L_{1}, L_{2}, L_{3}$ we have

$$
\begin{aligned}
& \frac{\partial}{\partial m_{3}^{2}} \text { pen }_{i j}^{(0)}=\frac{H_{0}^{\prime}(1+\epsilon)}{H_{1}} \text { pen }^{(-1)}-\frac{\left(A H_{0}^{\prime}+H_{1} B^{\prime}\right)}{2\left(A H_{0}+B H_{1}\right)} \text { pen }^{(0)}+\frac{(1+\epsilon)}{A H_{0}+B H_{1}}\left(-\frac{A H_{0}^{\prime}}{H_{1}}-B^{\prime}\right) \text { Box }^{(-1)} \\
& \quad+\frac{(1+\epsilon)}{2}\left(\frac{2 r_{3} W_{i ; i j}}{Z_{i}}+\frac{2 r_{1}}{\beta_{i} \beta_{j} Z_{i}\left(Z_{i}^{2}-Z_{j}^{2}\right)}+B^{\prime} \lambda_{i ; i j}+\frac{H_{0}^{\prime}\left(2 G_{i j} W_{i ; i j}+A Z_{i} \lambda_{i ; i j}\right)}{H_{1} Z_{i}}\right) \operatorname{Tri}^{(-1)}\left(Z_{i}\right) \\
& \quad+\frac{(1+\epsilon)}{2}\left(\frac{2 r_{3} W_{j ; i j}}{Z_{j}}+\frac{2 r_{1}}{\beta_{i} \beta_{j} Z_{j}\left(Z_{j}^{2}-Z_{i}^{2}\right)}+B^{\prime} \lambda_{j ; i j}+\frac{H_{0}^{\prime}\left(2 G_{i j} W_{j ; i j}+A Z_{j} \lambda_{j ; i j}\right)}{H_{1} Z_{j}}\right) \operatorname{Tri}^{(-1)}\left(Z_{j}\right) \\
& \quad+\left(\frac{H_{0}^{\prime} G_{i j} W_{0 ; i j}}{H_{1}}+r_{3} W_{0 ; i j}\right) \int_{0}^{1} d u u^{-1-\epsilon} \frac{1}{\sqrt{1-u}\left(H_{0}+H_{1} u\right)}
\end{aligned}
$$

In (5.38), the last term will be canceled when summing over three $\frac{\partial}{\partial m_{3}^{2}} p^{(0)} n_{i j}^{(0)}$ in (5.15). To continue, we need to reduce $\operatorname{Tr} i^{(-1)}, B o x^{(-1)}$ and $p e n^{(-1)}$ to our scalar basis. Using

$$
H_{0} \text { pen }^{(n)}+H_{1} \text { pen } n^{(n+1)}=B o x^{(n)}
$$

with $n=-1$,

$$
\operatorname{Tri}^{(-1)}(Z)=\frac{\epsilon}{(\epsilon+1)\left(1-Z^{2}\right)} \operatorname{Tri}^{(0)}(Z)+\frac{(1-2 \epsilon) Z}{\left(1-Z^{2}\right)(\epsilon+1)} B u b^{(0)}
$$

and

$$
\begin{aligned}
B o x^{(-1)}= & \frac{\frac{1}{2}+\epsilon}{1+\epsilon} \frac{A}{B} B o x^{(0)}+\frac{\epsilon}{1+\epsilon} \frac{C_{Z_{1}}}{B Z_{1}\left(1-Z_{1}^{2}\right)} \operatorname{Tri}^{(0)}\left(Z_{1}\right)+\frac{\epsilon}{1+\epsilon} \frac{C_{Z_{2}}}{B Z_{2}\left(1-Z_{2}^{2}\right)} \operatorname{Tri}^{(0)}\left(Z_{2}\right) \\
& +\frac{1-2 \epsilon}{1+\epsilon}\left(\frac{C_{Z_{1}}}{\left(1-Z_{1}^{2}\right) B}+\frac{C_{Z_{2}}}{\left(1-Z_{2}^{2}\right) B}\right) B u b^{(0)}
\end{aligned}
$$


we have

$$
\frac{\partial}{\partial m_{3}^{2}} \operatorname{pen}_{i j}^{(0)}=q_{i j ; 5} \operatorname{pen}_{i j}^{(0)}+q_{i j ; 4} B o x_{i j}^{(0)}+q_{i j ; Z_{i}} \operatorname{Tri}^{(0)}\left(Z_{i}\right)+q_{i j ; Z_{j}} \operatorname{Tri}^{(0)}\left(Z_{j}\right)+q_{i j ; 2} B u b^{(0)}
$$

with the coefficients

$$
\begin{aligned}
q_{i j ; 5}= & l_{i j ; 5}=-\frac{H_{0}^{\prime}(1+\epsilon)}{H_{0}}-\frac{\left(A_{i j} H_{0}^{\prime}+H_{1} B_{i j}^{\prime}\right)}{2\left(A_{i j} H_{0}+B_{i j} H_{1}\right)} \\
q_{i j ; 4}= & \frac{\frac{1}{2}+\epsilon}{1+\epsilon} \frac{A}{B} l_{i j ; 4}=\frac{A_{i j}\left(B_{i j} H_{0}^{\prime}-B_{i j}^{\prime} H_{0}\right)\left(\frac{1}{2}+\epsilon\right)}{B_{i j} H_{0}\left(A_{i j} H_{0}+B_{i j} H_{1}\right)} \\
q_{i j ; Z_{i}}= & l_{i j ; 4} \times \frac{\epsilon}{1+\epsilon} \frac{C_{Z_{i} ; i j}}{B_{i j} Z_{i}\left(1-Z_{i}^{2}\right)}+l_{i j ; Z_{i}} \times \frac{\epsilon}{(\epsilon+1)\left(1-Z_{i}^{2}\right)} \\
= & \frac{q_{i ; i j ; n}}{B_{i j} H_{0}\left(A_{i j} H_{0}+B_{i j} H_{1}\right) Z_{i}\left(Z_{i}^{2}-1\right)\left(H_{0}+H_{1}\left(1-Z_{i}^{2}\right)\right)\left(Z_{i}^{2}-Z_{j}^{2}\right) \beta_{i} \beta_{j}} \\
q_{i j ; Z_{j}}= & l_{i j ; 4} \times \frac{\epsilon}{1+\epsilon} \frac{C_{Z_{j} ; i j}}{B_{i j} Z_{j}\left(1-Z_{j}^{2}\right)}+l_{i j ; Z_{2}} \times \frac{\epsilon}{(\epsilon+1)\left(1-Z_{j}^{2}\right)} \\
= & \frac{q_{j ; i j ; n}}{B_{i j} H_{0}\left(A_{i j} H_{0}+B_{i j} H_{1}\right) Z_{j}\left(Z_{j}^{2}-1\right)\left(H_{0}+H_{1}\left(1-Z_{j}^{2}\right)\right)\left(Z_{j}^{2}-Z_{i}^{2}\right) \beta_{i} \beta_{j}} \\
q_{i j ; 2}= & l_{i j ; 4} \times \frac{1-2 \epsilon}{1+\epsilon}\left(\frac{C_{Z_{i} ; i j}}{\left(1-Z_{i}^{2}\right) B_{i j}}+\frac{C_{Z_{j} ; i j}}{\left(1-Z_{j}^{2}\right) B_{i j}}\right) \\
& +l_{i j ; Z_{i}} \times \frac{(1-2 \epsilon) Z_{i}}{\left(1-Z_{i}^{2}\right)(\epsilon+1)}+l_{i j ; Z_{j}} \times \frac{(1-2 \epsilon) Z_{j}}{\left(1-Z_{j}^{2}\right)(\epsilon+1)}
\end{aligned}
$$

where

$$
\begin{aligned}
& q_{i ; i j ; n}=\epsilon[ B^{2} H_{0}\left(-D H_{0}^{\prime}+2 D^{\prime} H_{1} Z_{i}^{2}+C H_{0}^{\prime}\left(1+Z_{i}^{2}\right)\right) \\
&+B^{\prime} C_{Z_{i}} H_{0}\left(H_{0}+H_{1}\left(1-Z_{i}^{2}\right)\right)\left(Z_{i}^{2}-Z_{j}^{2}\right) \beta_{i} \beta_{j} \\
&+B\left(A H_{0}\left(D H_{0}^{\prime}+\left(2 D^{\prime} H_{0}-2 D H_{0}^{\prime}\right) Z_{i}^{2}+C H_{0}\left(Z_{i}^{2}-1\right)\right)\right. \\
&+B^{\prime} H_{0}\left(D\left(H_{0}+H_{1}\left(1-2 Z_{i}^{2}\right)\right)-C\left(H_{0}+H_{1}+H_{1} Z_{i}^{2}-H_{1} Z_{i}^{2}\right)\right) \\
&\left.\left.\quad-C_{Z_{i}} H_{0}^{\prime}\left(H_{0}+H_{1}-H_{1} Z_{i}^{2}\right)\left(Z_{i}^{2}-Z_{j}^{2}\right) \beta_{i} \beta_{j}\right)\right] \\
& q_{j ; i j ; n}=\left.q_{j ; i j}\right|_{i \leftrightarrow j}, \quad B^{\prime} \equiv \frac{\partial B}{\partial m_{3}^{2}}, \quad D^{\prime} \equiv \frac{\partial D}{\partial m_{3}^{2}}, \quad H_{0}^{\prime} \equiv \frac{\partial H_{0}}{\partial m_{3}^{2}}, \quad C_{Z_{k} ; i j}=D_{i j}+\left(Z_{k}^{2}-1\right) C_{i j}
\end{aligned}
$$

Now we put the result (5.42) to (5.15) to get

$$
\begin{aligned}
& \frac{\partial}{\partial m_{3}^{2}} C\left(I_{5}\right)=\frac{b}{4\left(K^{2}\right)^{2}}\left\{\left(\frac{\partial T_{12}}{\partial m_{3}^{2}}+q_{12 ; 5} T_{12}\right) p e n_{12}^{(0)}+\left(\frac{\partial T_{13}}{\partial m_{3}^{2}}+q_{13 ; 5} T_{13}\right) p e n_{13}^{(0)}\right. \\
& +\left(\frac{\partial T_{23}}{\partial m_{3}^{2}}+q_{23 ; 5} T_{23}\right) p e n_{23}^{(0)}+q_{12 ; 4} T_{12} B o x_{12}^{(0)}+q_{13 ; 4} T_{13} B o x_{13}^{(0)} \\
& +q_{23 ; 4} T_{23} B_{o x}^{(0)}+\left(q_{12 ; Z_{1}} T_{12}+q_{13 ; Z_{1}} T_{13}\right) \operatorname{Tri}^{(0)}\left(Z_{1}\right) \\
& +\left(q_{12 ; Z_{2}} T_{12}+q_{23 ; Z_{2}} T_{23}\right) \operatorname{Tri}^{(0)}\left(Z_{2}\right)+\left(q_{13: Z_{3}} T_{13}+q_{23 ; Z_{3}} T_{23}\right) \operatorname{Tri}^{(0)}\left(Z_{3}\right) \\
& \left.+\left(q_{12 ; 2} T_{12}+q_{13 ; 2} T_{13}+q_{23 ; 2} T_{23}\right) B u b^{(0)}\right\}
\end{aligned}
$$


where $K=K_{1}$ and the parameters are given in (5.43) and (5.7). One important point is that the sum of the first three terms in (5.45) gives exactly the cut of pentagon in (5.11). Thus we have the result

$$
\begin{aligned}
I_{5}(1,1,1,1,2)= & c_{5 \rightarrow 5 ; K_{1}} I_{5}+c_{5 \rightarrow 4 ; \overline{5} ; K_{1}} I_{4 ; \overline{5}}+c_{5 \rightarrow 3 ; \overline{4} ; K_{1}} I_{4 ; \overline{4}}+c_{5 \rightarrow 4 ; \overline{3} ; K_{1}} I_{4 ; \overline{3}} \\
& +c_{5 \rightarrow 3 ; \overline{4} \overline{5} ; K_{1}} I_{3 ; \overline{4} \overline{5}}+c_{5 \rightarrow 3 ; \overline{3} \overline{5} ; K_{1}} I_{3 ; \overline{3} \overline{5}}+c_{5 \rightarrow 3 ; \overline{3} \overline{4} ; K_{1}} I_{3 ; \overline{3} \overline{4}}+c_{5 \rightarrow 2 ; \overline{3} \overline{4} \overline{5} ; K_{1}} I_{2 ; \overline{3} \overline{4} \overline{5}}+\cdots
\end{aligned}
$$

where the elips represents the tadpoles and the coefficients are given by

$$
\begin{aligned}
c_{5 \rightarrow 5 ; K_{1}}= & \frac{1}{T_{12}} \frac{\partial T_{12}}{\partial m_{3}^{2}}+q_{12}=\frac{1}{T_{13}} \frac{\partial T_{13}}{\partial m_{3}^{2}}+q_{13}=\frac{1}{T_{23}} \frac{\partial T_{23}}{\partial m_{3}^{2}}+q_{23} \\
c_{5 \rightarrow 4 ; \overline{3} ; K_{1}}= & \frac{1}{2 K^{2}} q_{23 ; 4} T_{23}, \quad c_{5 \rightarrow 4 ; \overline{4}}=\frac{1}{2 K^{2}} q_{13 ; 4} T_{13}, \quad c_{5 \rightarrow 4 \overline{5}}=\frac{1}{2 K^{2}} q_{12 ; 4} T_{12} \\
c_{5 \rightarrow 3 ; \overline{4} ; K_{1}}= & \frac{-b \sqrt{\Delta_{3 ; m=0\left[Z_{1}\right]}}\left(q_{12 ; Z_{1}} T_{12}+q_{13 ; Z_{1}} T_{13}\right), \quad \Delta_{3 ; m=0}\left[Z_{1}\right]=4\left[\left(K \cdot P_{1}\right)^{2}-K^{2} P_{1}^{2}\right]}{4 K^{4}}\left(q_{12 ; Z_{2}} T_{12}+q_{23 ; Z_{2}} T_{23}\right), \quad \Delta_{3 ; m=0}\left[Z_{2}\right]=4\left[\left(K \cdot P_{2}\right)^{2}-K^{2} P_{2}^{2}\right] \\
c_{5 \rightarrow 3 ; \overline{3} \overline{5} ; K_{1}}= & \frac{-b \sqrt{\Delta_{3 ; m=0\left[Z_{2}\right]}}}{4 K^{4}}\left(q_{13 ; Z_{3}} T_{13}+q_{23 ; Z_{3}} T_{23}\right), \quad \Delta_{3 ; m=0}\left[Z_{3}\right]=4\left[\left(K \cdot P_{3}\right)^{2}-K^{2} P_{3}^{2}\right] \\
c_{5 \rightarrow 3 ; \overline{3} ; K_{1}}= & \frac{-b \sqrt{\Delta_{3 ; m=0\left[Z_{3}\right]}}}{4 K^{4}}\left(P_{12}\right), \quad P_{2}=K_{123}, \quad P_{3}=K_{1234} \\
c_{5 \rightarrow 2 ; \overline{3} \overline{4} ; K_{1}}= & \left.\frac{1}{4 K^{4}}\left(q_{12 ; 2} T_{12}+q_{13 ; 2} T_{13}+q_{23 ; 2} T_{23}\right), \quad P_{1}=K_{12}, \quad P_{1}\right)
\end{aligned}
$$

with the parameters given in (5.43). The corresponding basis are

$$
\begin{aligned}
I_{4 ; \overline{5}} & =I_{5}(1,1,1,1,0), \quad I_{4 ; \overline{4}}=I_{5}(1,1,1,0,1), \quad I_{4 ; \overline{3}}=I_{5}(1,1,0,1,1) \\
I_{3 ; \overline{4} \overline{5}} & =I_{5}(1,1,1,0,0), \quad I_{3 ; \overline{3} \overline{5}}=I_{5}(1,1,0,1,0), \quad I_{3 ; \overline{3} \overline{4}}=I_{5}(1,1,0,0,1), \quad I_{2 ; \overline{3} \overline{4} \overline{5}}=I_{5}(1,1,0,0,0)
\end{aligned}
$$

Again, having presented details for the cut $K_{1}$, the computation of other cuts will be similar, as shown in the section of triangle. For simplicity we will not list them one by one. All coefficients have been checked using LiteRed [23] numerically.

\section{Conclusion}

In this paper, we have considered the reduction of one-loop integrals with higher poles using the unitarity cut method. By the trick of differentiation over auxiliary masses, we have translate the problem to the decomposition of differentiation of imaginary part of scalar basis. Furthermore, from the angle of differentiation, recurrence relation can be established and the whole reduction can be reduced to the reduction of basic integral, i.e., one and only one propagator with power two. We demonstrate our method by carrying out the reduction of basic example for the scalar bubbles, triangles, boxes and pentagons and give analytic expression for reduction coefficients.

One of unsolved problems of our algorithm is the analytic tadpole coefficients of the reduction. Although the recurrence relation can be established for the tadpole coefficients, the tadpole coefficients for the basic integrals, for examples $I_{2}(1,2), I_{3}(1,1,2), I_{4}(1,1,1,2)$ 
and $I_{5}(1,1,1,1,2)$ can not be found by unitarity cut method. One can use the technique developed in [19-21] or the familiar IBP method to calculate them to complete the whole reduction program for higher poles.

\section{Acknowledgments}

We would like to thank Y.Zhang for very useful discussion and reading of draft. This work is supported by Qiu-Shi Funding and Chinese NSF funding under Grant No.11935013, No.11947301, No.12047502 (Peng Huanwu Center).

Open Access. This article is distributed under the terms of the Creative Commons Attribution License (CC-BY 4.0), which permits any use, distribution and reproduction in any medium, provided the original author(s) and source are credited.

\section{References}

[1] Z. Bern, L.J. Dixon, D.C. Dunbar and D.A. Kosower, One loop n point gauge theory amplitudes, unitarity and collinear limits, Nucl. Phys. B 425 (1994) 217 [hep-ph/9403226] [INSPIRE].

[2] Z. Bern, L.J. Dixon, D.C. Dunbar and D.A. Kosower, Fusing gauge theory tree amplitudes into loop amplitudes, Nucl. Phys. B 435 (1995) 59 [hep-ph/9409265] [INSPIRE].

[3] R. Britto, F. Cachazo and B. Feng, Generalized unitarity and one-loop amplitudes in $N=4$ super-Yang-Mills, Nucl. Phys. B 725 (2005) 275 [hep-th/0412103] [INSPIRE].

[4] F. Cachazo, P. Svrček and E. Witten, Twistor space structure of one-loop amplitudes in gauge theory, JHEP 10 (2004) 074 [hep-th/0406177] [INSPIRE].

[5] R. Britto, E. Buchbinder, F. Cachazo and B. Feng, One-loop amplitudes of gluons in SQCD, Phys. Rev. D 72 (2005) 065012 [hep-ph/0503132] [INSPIRE].

[6] R. Britto, B. Feng and P. Mastrolia, The cut-constructible part of QCD amplitudes, Phys. Rev. D 73 (2006) 105004 [hep-ph/0602178] [INSPIRE].

[7] C. Anastasiou, R. Britto, B. Feng, Z. Kunszt and P. Mastrolia, D-dimensional unitarity cut method, Phys. Lett. B 645 (2007) 213 [hep-ph/0609191] [InSPIRE].

[8] C. Anastasiou, R. Britto, B. Feng, Z. Kunszt and P. Mastrolia, Unitarity cuts and Reduction to master integrals in d dimensions for one-loop amplitudes, JHEP 03 (2007) 111 [hep-ph/0612277] [INSPIRE].

[9] R. Britto and B. Feng, Unitarity cuts with massive propagators and algebraic expressions for coefficients, Phys. Rev. D 75 (2007) 105006 [hep-ph/0612089] [InSPIRE].

[10] R. Britto and B. Feng, Integral coefficients for one-loop amplitudes, JHEP 02 (2008) 095 [arXiv:0711.4284] [INSPIRE].

[11] R. Britto, B. Feng and P. Mastrolia, Closed-Form Decomposition of One-Loop Massive Amplitudes, Phys. Rev. D 78 (2008) 025031 [arXiv:0803.1989] [InSPIRE].

[12] R. Britto, B. Feng and G. Yang, Polynomial Structures in One-Loop Amplitudes, JHEP 09 (2008) 089 [arXiv:0803.3147] [INSPIRE]. 
[13] B. Feng and H. Wang, Analytic structure of one-loop coefficients, JHEP 05 (2013) 104 [arXiv: 1301.7510] [INSPIRE].

[14] M. Sogaard and Y. Zhang, Unitarity Cuts of Integrals with Doubled Propagators, JHEP 07 (2014) 112 [arXiv:1403.2463] [INSPIRE].

[15] J.-H. Zhang, Multidimensional Residues for Feynman Integrals with Generic Power of Propagators, arXiv:1112.4136 [INSPIRE].

[16] S. Abreu, F. Febres Cordero, H. Ita, M. Jaquier and B. Page, Subleading Poles in the Numerical Unitarity Method at Two Loops, Phys. Rev. D 95 (2017) 096011 [arXiv: 1703.05255] [INSPIRE].

[17] G. Passarino and M.J.G. Veltman, One Loop Corrections for $e^{+} e^{-}$Annihilation Into $\mu^{+} \mu^{-}$ in the Weinberg Model, Nucl. Phys. B 160 (1979) 151 [INSPIRE].

[18] A. Primo and L. Tancredi, On the maximal cut of Feynman integrals and the solution of their differential equations, Nucl. Phys. B 916 (2017) 94 [arXiv:1610.08397] [InSPIRE].

[19] R. Britto and B. Feng, Solving for tadpole coefficients in one-loop amplitudes, Phys. Lett. B 681 (2009) 376 [arXiv:0904.2766] [INSPIRE].

[20] R. Britto and E. Mirabella, Single Cut Integration, JHEP 01 (2011) 135 [arXiv:1011.2344] [INSPIRE].

[21] R. Britto and E. Mirabella, Massive particles and unitarity cuts, in International Workshop on Future Linear Colliders (LCWS11), (2012) [arXiv:1202.2426] [INSPIRE].

[22] V.A. Smirnov, Evaluating Feynman Integrals, Springer Berlin Heidelberg (2005), [DOI].

[23] R.N. Lee, LiteRed 1.4: a powerful tool for reduction of multiloop integrals, J. Phys. Conf. Ser. 523 (2014) 012059 [arXiv: 1310.1145] [INSPIRE]. 